\title{
虚拟随机车路场下驾驶人驾驶能力机理分析
}

\author{
孙博华 ${ }^{1}$ 邓伟文 ${ }^{1,2}$ 吴 坚 ${ }^{1}$ 李雅欣 $^{1}$ \\ (1. 吉林大学汽车仿真与控制国家重点实验室 长春 130022; \\ 2. 北京航空航天大学交通科学与工程学院 北京 430071)
}

\begin{abstract}
摘要: 为解决智能汽车人机协同共驾驾驶权仲裁的普适性和规模可控性问题, 提出虚拟随机车路场下的驾驶人驾驶能力机理 分析及评估体系。作为适应于驾驶人驾驶能力机理分析的评估环境, 虚拟随机车路场模型通过耦合车辆运动模型及车路可行 驶区域空间拓扑结构的时空状态, 得到可揭示微观驾驶场景下人-车-路耦合机理及车路协同规律的驾驶场景。驾驶能力机理 分析体系在严格定义驾驶能力的基础上, 建立满足高阶非线性驾驶能力属性的离线辨识模型。模型中关键参数经解耦和降维 组成的样本集, 为表征驾驶能力内在属性的关键依据。采用主客观相结合方式分类驾驶能力, 采用基于混合高斯隐马尔科夫 过程实现驾驶能力的实时辨识。结果表明, 所提出的虚拟随机车路场模型可以客观地反映交通流的波粒二象性, 通过虚拟随 机车路场下的驾驶能力机理分析, 可以得到准确且可靠的驾驶能力评价结果。虚拟随机车路场下的驾驶人驾驶能力机理分析 体系，优化了驾驶权仲裁机制的合理性，提高了人机协同共驾系统的安全性和驾驶人可接受性。
\end{abstract}

关键词: 车辆工程; 随机车路场; 驾驶能力; 机器学习; 系统辨识

中图分类号: U461

\section{Mechanism Analysis of Driving Capability in the Virtual Random Vehicle-road Field}

\author{
SUN Bohua ${ }^{1}$ DENG Weiwen ${ }^{1,2}$ WU Jian $^{1} \quad$ LI Yaxin $^{1}$ \\ (1. State Key Laboratory of Automotive Simulation and Control, Jilin University, Changchun 130022; \\ 2. School of Transportation Science and Engineering, Beihang University, Beijing 430071)
}

\begin{abstract}
To solve the universality and scale controllability issue of the driving authority arbitration in shared control, an analysis and evaluation framework of the driving capability mechanism in the virtual random vehicle-road field(RVRF) is proposed. As the assessment environment for the mechanism analysis of the driving capability, driving scenarios those can reveal the human-vehicle-road coupling mechanism and vehicle-road cooperative rules by coupling the space topology of drivable area with the vehicle motion patterns are obtained in the RVRF. Basing on the strict definition of driving capability, the off-line identification model satisfies the high-order nonlinear attributes of driving capability is established in the RVRF evaluation framework. The sample set consisting of the decoupled and dimensionless key parameters in the model is the key basis to represent the intrinsic attribute of driving capability. The classification is done basing on the combination of subjective and objective and driving capability is identified in real time basing on Gaussian multi-dimension hidden markov model(GM-HMM). Results show that the proposed virtual RVRF can objectively reflect the wave-particle duality of traffic flow and the proposed evaluation method for driving capability in the virtual RVRF can achieve accurate and reliable evaluation results. The mechanism analysis of driving capability in the virtual RVRF optimizes the rationality of driving authority arbitration mechanism, and improves the safety and driver acceptability for the shared control system.
\end{abstract}

Key words: vehicle engineering; random vehicle-road field; driving capability; machine learning; system identification

\section{0 前言}

以人机协同共驾为代表的智能车辆和智能交通 系统需要加强对驾驶人行为模式的理解。深刻揭示

* 国家重点研发计划(2016YFB0100904)和国家自然科学基金(U1564211, 51775235)资助项目。20191201 收到初稿, 20200318 收到修改稿
驾驶人行为模式机理，可以对智能汽车人机协同共 驾驾驶权进行合理分配，充分发挥人机优势，进而 提高智能车辆的行驶安全性和驾乘舒适性。因此, 日益受到各国学者的广泛关注 ${ }^{[1-2]}$ 。

建立合理的驾驶权分配机制是实现人机协同共 驾友好交互的关键。SOUALMI 等 ${ }^{[3]}$ 在人机协同共驾 控制器中加入驾驶人状态监测模块, 进而帮助上层 
控制器决策人机驾驶权。ANDERSON 等 ${ }^{[4]}$ 基于自然 驾驶汇流场景评估, 实现人机协同跟车控制。谈东 奎 ${ }^{[5]}$ 建立驾驶人转向技能模型的轨迹预测方式, 完 成人机协同控制的驾驶权决策。表明探索驾驶人行 为模式中的驾驶状态、驾驶习性及驾驶技能对于建 立合理的驾驶权分配机制具有重要意义。赵治国 等 ${ }^{[6]}$ 通过驾驶人预瞄模型实现特定驾驶技能下的 “类人” 无人驾驶。GROOT 等 ${ }^{[7]}$ 通过在模拟器上配 置不同的轮胎-路面摩擦系数, 进而分析了驾驶技能 与驾驶习性的耦合关系和影响因素。SUN 等 ${ }^{[8]}$ 基于 Hammerstein 模型解耦驾驶习性与驾驶技能。表明 驾驶状态具有强随机性和复杂多变性, 且分别与驾 驶习性和驾驶技能问呈弱耦合关系, 驾驶习性及驾 驶技能彼此呈强耦合关系。

通过建立驾驶人驾驶行为模式的分类、辨识及 表征等评价体系, 可以揭示驾驶行为模式的一般规 律, 一般可以通过模式识别或系统辨识等基础学科 理论解决此类问题。MARTINEZ 等 ${ }^{[9]}$ 总结了目前驾 驶习性的分类及辨识方法, 并阐述未来趋势集中于 更大数据集下驾驶习性多元化研究, 以及实现基于 有/无监督的结合算法增强驾驶人的适应性和认知 能力。MARS 等 ${ }^{[10]}$ 通过建立基于控制论的驾驶人转 向辨识模型, 进而在不同驾驶注意力分散状态下对 驾驶状态进行估计。综上可知, 采用基于系统辨识 或机器学习的驾驶行为辨识模型, 有助于实现驾驶 行为模式处于黑箱时的辨识; 可以采用主客观评价 方法, 得到接近其真值的驾驶行为模式分类结果。

安全、高效且智能的驾驶人驾驶行为模式数据 采集及测试环境, 对驾驶人行为模式机理分析结果 具有重要影响。许力等 ${ }^{[11]}$ 建立起面向大数据训练的 个性化驾驶人模型。ZAVALKO ${ }^{[12]}$ 通过在自然驾驶 环境下大规模采集商用车驾驶人油耗数据, 进而辨 识出环保型驾驶人, 并将其驾驶数据用于控制器中 节能功能的开发。刘明周等 ${ }^{[13]}$ 通过机器视觉信息提 取驾驶人眼、嘴及手部特征, 进而推断驾驶人疲劳 程度。综上可知, 现有驾驶人行为模式数据采集及 测试方法主要通过自然驾驶工况和虚拟环境下的理 想激励工况手段完成。自然驾驶工况下的数据采集、 测试耗时和工况匹配的效率低且不适用于危险驾驶 行为模式, 而虚拟环境下的激励工况多为人为规定 的单车或理想激励工况, 由此采集的驾驶人数据在 很大程度上无法真实反映驾驶人驾驶行为模式。建 立面向驾驶人行为模式数据采集及测试用虚拟交通 场景, 通过客观反映人-车-路耦合机理及车路协同 规律, 可以快速而安全地采集更具客观性的驾驶行 为模式数据。
自然驾驶场景中人-车-路系统为非线性、多重 反馈且复杂时变系统, 故一般可以通过系统动力学 或机器学习等基础学科理论模拟自然交通场景的演 化规律 ${ }^{[14-15]}$ 。ZHANG 等 ${ }^{[16]}$ 采通过建立带有时滞速 度差的跟车动力学模型进而揭示时滞速度对车路流 的影响。HUANG 等 ${ }^{[17]}$ 通过深度自学习网络及多层 回归方法克服了传统交通流建模时模型简单及主观 建模错误等缺陷。BORSCHE 等 ${ }^{[18]}$ 通过建立微观宏观交通流模型进而揭示带有行人的交叉口人-车路流模型。结果表明, 采用面向数据学习的自然交 通场景建模方式, 有助于避免主观建模错误或模型 过于简单等缺陷; 采用微观-宏观一体化建模框架, 可以更客观地反应微观及宏观自然交通场景的波粒 二象性，进而获得更具一致性的虚拟交通场景。

本文通过建立虚拟随机车路场，同步提高了驾 驶人驾驶行为模式数据采集及测试效率、安全性及 客观性; 通过分析虚拟随机车路场下驾驶人驾驶能 力机理, 进而解决了驾驶人行为模式影响因素问耦 合多变特性带来的人机协同共驾驾驶权分配机制合 理性问题。首先建立了微观车路场模型并分类处车 路场空间态势, 通过耦合车路的时空状态建立起虚 拟随机车路场模型。对驾驶能力概念提出的意义进 行了描述和分析, 对驾驶人驾驶能力进行了定义, 在搭建的驾驶人在环智能仿真平台上的虚拟随机车 路场中进行数据采集。进而分析驾驶能力内在属性 和表征方法。建立驾驶能力辨识模型并采用主成分 分析法对模型进行解耦合降维, 通过客观聚类方法 实现驾驶能力的分类。最终通过混合高斯模型实时 辨识驾驶人的驾驶能力。

\section{1 概念描述及数据采集}

\section{1 自然驾驶场景的场论化描述}

车-车协同博栾耦合关系及车-路协同约束耦合 关系普遍存在于各种自然驾驶场景中, 使自然驾驶 场景下的交通流在时空维度上出现动态、连续及部 分可观测性。场作为物质存在的基本形式之一，具 有时空维度上的连续变化特性及可测性。故可以采 用场论建立虚拟车路场模型, 进而揭示人-车-路耦 合机理及车路协同规律。设虚拟车路场 $E_{v}$ 由运动物 体组成的动能场 $E_{k}$ 、静止物体组成的势能场 $E_{p}$ 及驾 驶人因不确定性带来的意图场 $E_{i}$ 等三部分组成, 如 式(1)所示

$$
E_{v}=E_{k}+E_{p}+E_{i}
$$

受到王建强等 ${ }^{[19]}$ 对行车风险场研究的启发, 交 
通参与物 $P$ 在空间点 $\left(x_{q}, y_{q}\right)$ 处产生的 $E_{k} 、 E_{p}$ 及 $E_{i}$ 按式(2)所示

$$
\begin{gathered}
E_{k}=\frac{G R_{p} M_{p}}{\left|r_{q}\right|^{k_{1}}} \frac{r_{q}}{\left|r_{q}\right|} \exp \left(k_{2} v_{p} \cos \left(\theta_{p}\right)\right) \\
E_{p}=\frac{G R_{p} M_{p}}{\left|r_{q}\right|^{k_{1}}} \frac{r_{q}}{\left|r_{q}\right|} \\
E_{i}=\frac{G R_{p} M_{p}}{\left|r_{q}\right|^{k_{1}}} \frac{r_{q}}{\left|r_{q}\right|} \exp \left(k_{2} v_{p} \cos \left(\theta_{p}\right)\right) \Phi_{D}
\end{gathered}
$$

式中, $G 、 k_{1} 、 k_{2}$ 均为待定常数, $v_{p}$ 为交通参与物 $P$ 的纵向车速, $r_{q}$ 为交通参与物 $P$ 到点 $\left(x_{q}, y_{q}\right)$ 处的距离 矢量, $\Phi_{D}$ 为意图不确定因数。 $M_{p}$ 为交通参与物的等 效质量, $R_{P}$ 为道路场指数, $M_{p}$ 及 $R_{p}$ 如式(5)所示

$$
\begin{gathered}
M_{p}=T_{p} m_{p}\left(1+\sum_{k} \alpha_{k} v_{p}^{\beta_{k}}\right) \\
R_{p}= \begin{cases}\left(\frac{\delta_{p}}{\delta_{s}}\right)^{\gamma_{1}} & v_{p}=0 \\
\left(\frac{\delta_{p}}{\delta_{s}}\right)^{\gamma_{1}}\left(\frac{\mu_{p}}{\mu_{s}}\right)^{\gamma_{2}} \exp \left(\rho_{p}^{\gamma_{3}}+\tau_{p}^{\gamma_{4}}\right) & v_{p} \neq 0\end{cases}
\end{gathered}
$$

式中, $T_{p}$ 为交通参与物 $P$ 的类型， $(\alpha, \beta)$ 为待定常 数, $k$ 为多项式项数, $\delta_{p}$ 为道路能见度, $\delta_{s}$ 为理想光 照下道路能见度, $\mu_{p}$ 为路面附着系数, $\mu_{s}$ 为高附着 路面附着系数, 取 $0.85,\left(\gamma_{1}, \gamma_{2}, \gamma_{3}, \gamma_{4}\right)$ 为待定常数, $\rho_{p}$ 为道路曲率, $\tau$ 为路面坡度。

以虚拟车路场为基础, 将虚拟随机车路场 (Random vehicle and road field, RVRF) 定义为虚拟仿 真环境下车-车及车-路彼此耦合形成的强随机稳态 场, 是虚拟环境下对自然驾驶场景中车-车及车-路 作用关系的精细模型, 在微观驾驶场景中具有强随 机粒子特性且在宏观场景中具有波运动特性。

\section{2 驾驶能力描述}

驾驶行为模式关键元素的耦合关系以及基于驾 驶行为模式的人-车-环境系统如图 1 所示。驾驶人 在交通状态、道路状态及本车状态的激励下, 基于 自身的驾驶习性、驾驶技能和驾驶状态产生了特定 的驾驶意图, 进而维持或改变本车状态。驾驶习性 与驾驶技能呈强耦合关系, 且分别与驾驶状态呈弱 耦合关系。在目前人机协同共驾驾驶权分配机制中, 驾驶习性、驾驶技能及驾驶状态作为彼此独立因素 考虑, 如式(7)所示

$$
W_{D}=f\left(D_{y}, D_{1}, D_{u}\right)
$$

式中, $D_{y}$ 为驾驶习性, $D_{1}$ 为驾驶技能, $D_{u}$ 为驾驶状 态。显然, 对于驾驶行为模式中关键元素的强制解
耦会降低驾驶权分配精度。可将 $D_{u}$ 定义为典型驾驶 状态的集合, 如式(8)所示

$$
D_{u}=\left\{d_{u, 1}, d_{u, 2}, \cdots, d_{u, m}\right\}
$$

式中, $d_{u}$ 为驾驶时注意力分散、疲劳、情绪波动等 驾驶状态子模型, $m$ 代表驾驶状态数目。每一个驾 驶状态分别对应一种驾驶人隐动作行为

$$
O_{u}=\left\{o_{u, 1}, o_{u, 2}, \cdots, o_{u, g}\right\}
$$

式中, $O_{u}$ 为驾驶人隐动作行为集合, $g$ 代表在特定 驾驶动作诱因中, 驾驶人隐动作行为集合数目。设 在全部交通-道路-本车系统中，驾驶动作诱因集合 为

$$
C_{u, j}=\left\{C_{u, k}, k=1,2, \cdots, j\right\}
$$

式中, $C_{u, k}$ 为驾驶动作诱因集合, $k$ 代表驾驶动作诱 因数目。则驾驶人处于任意 $C_{u, k}$ 时, 均对应一组 $O_{u}$, 记为 $C_{u, k} \rightarrow O_{u, k}$ 。可得到所有交通-道路-本车状态下 驾驶状态集合为

$$
D_{u}=\sum_{k=1}^{j} O_{u, g}
$$

显然, 在所有交通-道路-本车系统状态中, 驾 驶动作诱因的个数 $j$ 趋近于无穷，则 $C_{u, j}$ 无上界， 对应 $O_{u, k} \rightarrow \infty$, 即当 $j \rightarrow \infty, D_{u} \rightarrow \infty$ 。面向驾驶权分 配机制的驾驶状态会出现随交通-道路-本车系统状 态的多变性而无限延展的情况。

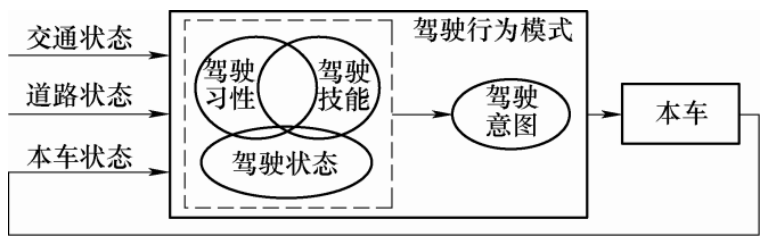

图 1 驾驶行为模式关键元素耦合关系

为提高驾驶权分配精度, 提出驾驶能力概念; 为提高驾驶权分配的普适性和规模可控性, 建立驾 驶能力的分类、辨识及表征评价体系。将驾驶能力 定义为驾驶人随外界环境负荷变化而渐变的对车辆 把控的能力, 是个人驾驶习性、驾驶技能及驾驶状 态的综合体，具有时变非线性动态特性；在评价手 段上, 通过采集本车及交通环境的物理状态信号, 对驾驶能力机理进行表征和评价。

\section{2 数据采集平台}

\section{1 自然交通场景实车平台}

\subsection{1 平台配置}

为采集自然交通场景下的车路数据, 搭建自然 交通场景实车平台, 如图 2 所示。型号 VN1640 的 
Vector 总线采集设备和 dSPACE MicroAutoBox 原型 控制器同时通过 CAN 总线采集型号 RT3002 惯导、 型号 ibeo LUX-4 四线激光雷达、型号 Delphi ESR 毫米波雷达及车载 CAN 总线信号, 防止数据丢失。 PC 机接收 Vector、MABX 及型号 scA1300-32 Basler 相机数据。

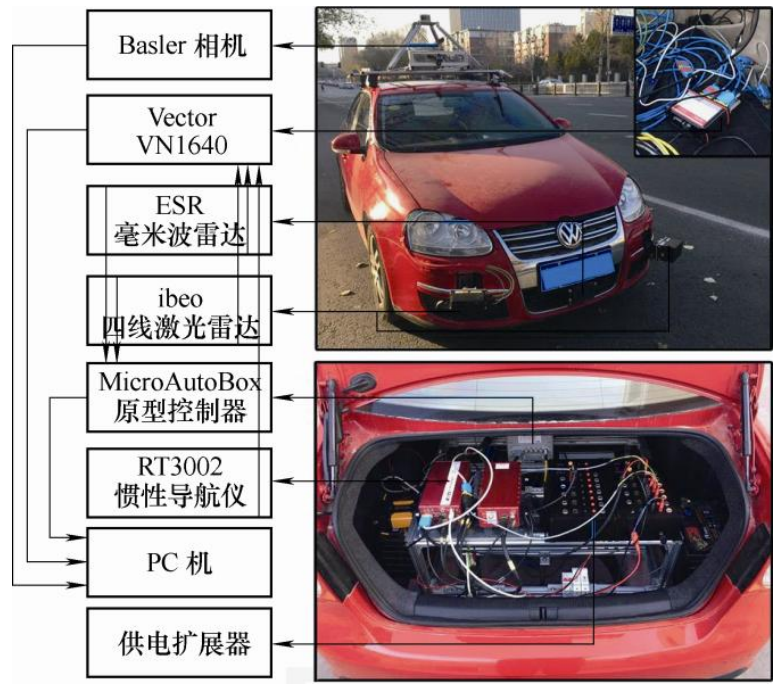

图 2 自然交通场景实车平台

RT3002 惯导用于测量车辆状态及位置, 带有 GPS 基站补偿的位置精度可达到 $1 \mathrm{~cm}$ 。 ibeo LUX-4 激光雷达探测角度为 $85^{\circ}$, 最大探测距离 $50 \mathrm{~m}$, 在 $12.5 \mathrm{~Hz}$ 扫描频率下的分辨率为 $0.25^{\circ}$ 。ESR 毫米波 雷达探测角度为 $90^{\circ}$, 最大探测距离 $60 \mathrm{~m}, 76$ $77 \mathrm{GHz}$ 扫描频率下的分辨率为 $\pm 0.5^{\circ}$, 将两个四线 激光雷达安装于车辆左右角点, 将 ESR 毫米波雷达 安装于车辆正前方, 通过目标融合方式可以实现 $210^{\circ}$ 感知角度及最大外包络 $50 \mathrm{~m}$ 的感知距离。 Basler 相机采集到的视频信息, 用于数据采集后处 理时的匹配和复现。采样周期为各传感器采样时间 的最大值。

\subsection{2 数据采集方案}

为保证虚拟 RVRF 的模型训练质量, 实现高逼 真度的自然交通场景模拟，选择 30 位年龄在 25 40 周岁、驾龄在 5 10 年且周驾驶时长不低于 $14 \mathrm{~h}$ 的驾驶人作为被测对象。各驾驶人在天气及路面状 况良好的自然交通中驾驶, 如图 3 所示。驾驶路线 由长春市内的若干主干道路包络为三块矩形区域, 分别采集三块矩形区域内全部道路下的自然驾驶数 据。采集的自然交通场景数据如表 1 所示，包含主 路及框内所有支路路段。各驾驶人在相同道路拓扑 结构对应的采集工况下需重复驾驶 10 次, 全部驾驶 人共采集约 3 万自然交通场景, 典型自然交通场景 数据片段共计约 66600 段。

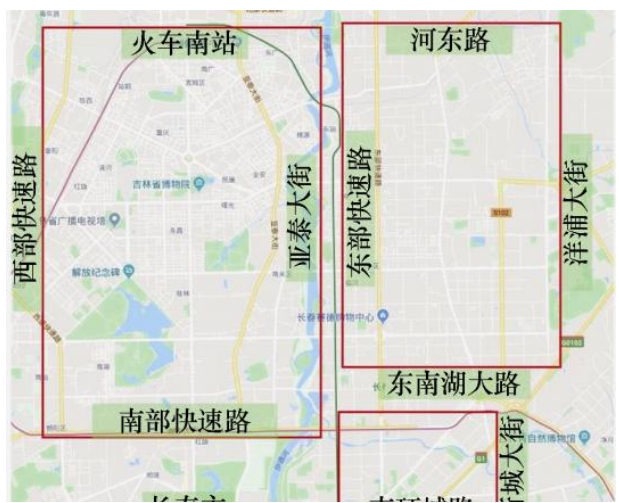

图 3 自然交通场景采集路线

表 1 自然交通场景采集工况

\begin{tabular}{|c|c|c|c|}
\hline 工况类型 & 一级子工况 & 二级子工况 & 主车路线 \\
\hline 直路 & 单/双/三/多车道 & \multirow{5}{*}{$\begin{array}{l}\text { (1) 有无道路 } \\
\text { 侧方停车; } \\
\text { (2) 周围交通 } \\
\text { 车密度稀疏/ } \\
\text { 正常/椆密 }\end{array}$} & \\
\hline 弯路 & $\begin{array}{l}\text { (1)小/中/大弯路 } \\
\text { (2)单至多车道 }\end{array}$ & & $\begin{array}{l}\text { (1)单车道上行驶; } \\
\text { (2)靠路沿石车道行驶; }\end{array}$ \\
\hline $\mathrm{T}$ 形路 & $\begin{array}{l}\text { (1)直角/非直角 } \\
\text { (2)单至三车道 }\end{array}$ & & (4)靠相向车行驶车道 \\
\hline 交叉口 & 有/无交通灯 & & \\
\hline 环岛 & $\begin{array}{l}\text { (1)大环岛/小环岛; } \\
\text { (2)进/出/绕行环岛 }\end{array}$ & & $\begin{array}{l}\text { (1)里圈/中圈/外圈 } \\
\text { (2)最里圈到最外圈 } \\
\text { (3)最外圈到最里圈 } \\
\text { (4)里圈到中圈到里圈 }\end{array}$ \\
\hline
\end{tabular}

\section{2 驾驶人在环智能模拟平台}

\subsection{1 平台配置}

为获得虚拟 RVRF 下驾驶人的驾驶能力数据, 同时确保试验的安全性和可操作性, 基于 dSPACE Simulator 实时仿真系统和 PanoSim 汽车智能驾驶仿 真系统建立驾驶人在环智能模拟平台, 如图 4 所示。
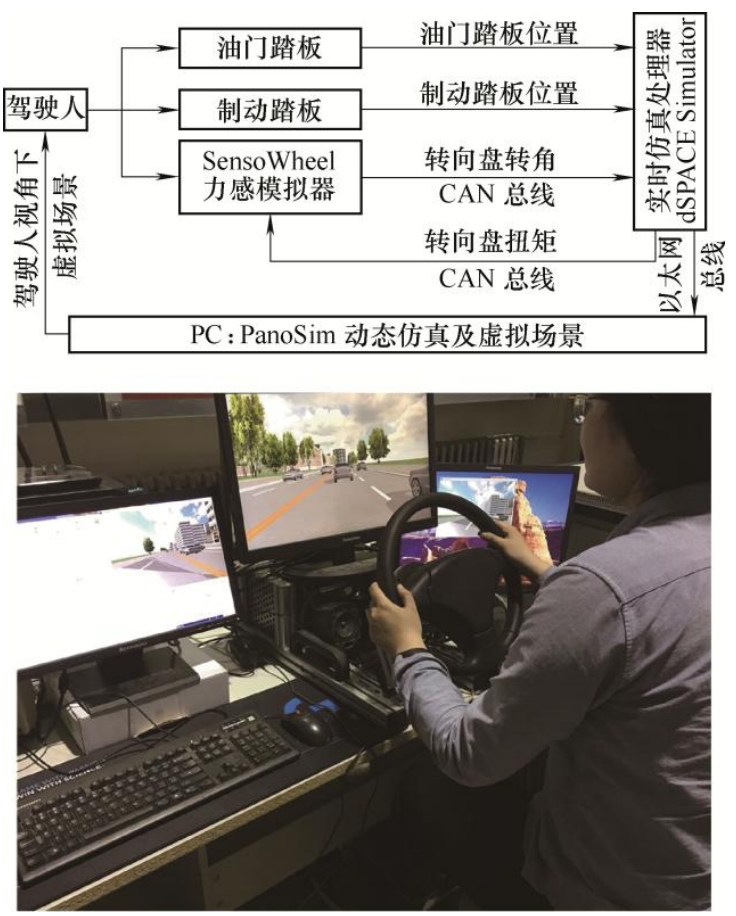

图 4 驾驶人在环智能仿真平台 
PanoSim 汽车智能驾驶仿真平台是本团队自主 研发的集车辆动力学、三维行驶环境、交通和车载 环境传感模型等于一体的大型汽车模拟仿真软件平 台, 可实现高精度的车辆动力学模拟、道路与场景 模拟、交通车辆模拟、雷达与相机模拟等, 支持驾 驶人在环仿真, 便于进行驾驶能力数据采集。选用 dSPACE DS1006 系统作为实时仿真模型处理器, 并 实时接收由真实加速踏板、制动踏板和 SensoWheel 力矩方向盘输入的真实驾驶人操纵信号, 同时反馈 转向盘扭矩至力矩转向盘。据此实时运行包含虚拟 随机车路场驾驶场景的 PanoSim 软件模型, 可以得 到主车状态、随机场路场交通密度及波动统计量、 随机车路场中其他交通参与物状态及其与主车的相 对状态信息。

\subsection{2 虚拟 RVRF 下典型激励场景配置}

为深入探究驾驶能力机理, 进而提取其内在属 性、表征与评价方法, 在虚拟 RVRF 下提取包含直 路、弯路、 $\mathrm{T}$ 形路、交叉口及环岛等典型道路拓扑 结构下的 RVRF 激励场景。选择 5 位年龄在 $25 \sim 40$ 周岁且驾龄在 $2 \sim 10$ 年驾驶人作为被测对象。将每 种典型道路拓扑结构对应的 RVRF 激励场景作为一 类, 驾驶人需在各类场景中反复不间断进行循环试 验, 各循环试验时长至少 $6 \mathrm{~h}$, 共需 10 组循环试验。 单次场景测试用时控制为 $[10,60] \mathrm{s}$ 区问, 驾驶人在 特定循环试验中连续 4 次单次激励场景下出现交通 事故或驾驶人主观表示无法继续驾驶, 则测试结束。 在试验进行过程中，随机采用与驾驶人交谈或使驾 驶人接听电话等扰动方式干扰驾驶人。

\section{3 虚拟 RVRF 中驾驶能力机理分析}

\section{1 虚拟 RVRF 中驾驶能力机理分析逻辑框架}

虚拟 RVRF 环境中驾驶能力机理分析逻辑框架 如图 5 所示。基于虚拟车路场模型将自然交通场景 中采集到的数据进行时间切片分析, 采用场图谱相 似性理论对时间切片后的虚拟车路场模型片段进行 聚类，得到典型的车路场空间态势。将典型车路场 空间态势对应的主车数据进行统计, 并基于生长型 神经气算法提取车路可行驶区域空问拓扑结构，同 时建立基于高斯过程回归的车辆运动模型。通过耦 合可行驶区域空间状态和车辆运动模型的时间状态, 建立起虚拟随机车路场模型。将驾驶能力表示为 $[0$, 1]区问上的连续值, 零值对应最差驾驶能力, 1 值对 应最佳驾驶能力。将虚拟 RVRF 中采集的驾驶能力 数据分别输入进基于 Hammerstein 模型的纵侧向驾 驶能力辨识模型中, 经过模型训练可得到辨

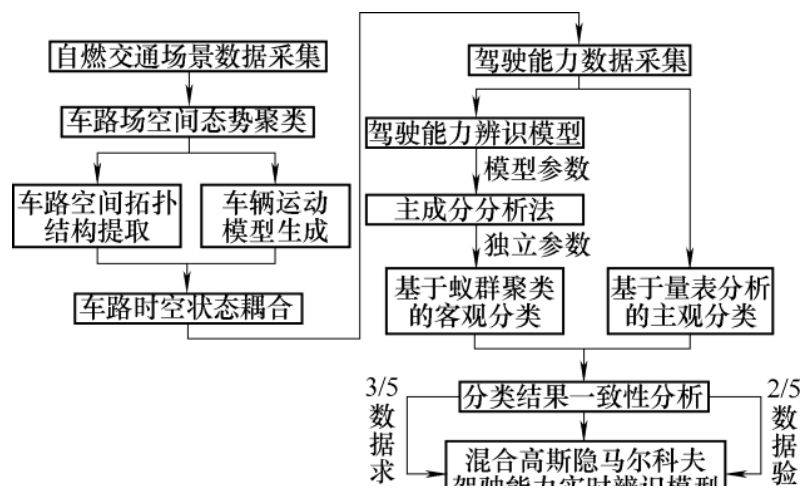

图 5 驾驶能力评价体系逻辑框架

识模型静态及动态环节的模型参数。模型参数经过 主成分分析, 得到解耦及降维的独立参数表达形式。 将独立参数输入客观分类中, 选取主客观分类结果 中相一致部分作为驾驶能力的真值。采用混合高斯 隐马尔科夫模型实时辨识驾驶人的驾驶能力。采用 部分驾驶能力真值数据训练驾驶能力实时辨识模型, 另一部分用于验证该模型的准确率。

\section{2 虚拟 RVRF}

\section{2 .1 车路场空间态势聚类}

将自然交通场景中采集到的数据进行时间切片, 得到特定时刻下虚拟车路场图谱如式(12)所示

$$
\Omega=\left\{E_{s, \zeta}\left(x_{\zeta}, y_{\zeta}\right), \zeta=1,2, \cdots, n\right\}
$$

式中, $E_{s, \zeta}$ 为特定时刻下车路场 $\left(x_{\zeta}, y_{\zeta}\right)$ 处场强, $\Omega$ 即 为特定时刻下虚拟车路场各坐标处场强组成的场图 谱。因此, 车路场空间态势聚类的过程即为提取场 图谱的统计特性并聚类场图谱的过程。

考虑到场图谱场强的空问变化特性及场强的邻 域相关性, 故采用均值邻域二维直方图理论提取场 图谱的统计特性。以 $\left(x_{\zeta}, y_{\zeta}\right)$ 坐标点为中心 $N \times N$ 窗 口邻域的平均场强, 如式(13)所示

$$
\begin{gathered}
G_{E}\left(x_{\zeta}, y_{\zeta}\right)=\frac{1}{N \times N} \sum_{i=(N-1) / 2}^{(N-1) / 2} \sum_{j=(N-1) / 2}^{(N-1) / 2} E_{s, \zeta}\left(x_{\zeta}+i, y_{\zeta}+j\right) \\
x_{\zeta} \in\left[1, Q_{x}\right] \quad y_{\zeta} \in\left[1, Q_{y}\right]
\end{gathered}
$$

式中, $G_{E}\left(x_{\zeta}, y_{\zeta}\right)$ 为 $\left(x_{\zeta}, y_{\zeta}\right)$ 坐标处的邻域平均场强, $Q_{x}$ 和 $Q_{y}$ 分别为场图谱的纵侧向边界。场图谱的均 值邻域二维直方图为 $\left(E_{s, \zeta}\left(x_{\zeta}, y_{\zeta}\right)=E_{m}, G_{E}\left(x_{\zeta}, y_{\zeta}\right)=E_{n}\right)$ 出现的概率, 如式(14)所示

$$
\begin{gathered}
H_{m}\left(E_{m}, E_{n}\right)=\mathrm{P}\left(E_{s, \zeta}\left(x_{\zeta}, y_{\zeta}\right)\right)= \\
\frac{N\left(E_{s, \zeta}\left(x_{\zeta}, y_{\zeta}\right)=E_{m}, G_{E}\left(x_{\zeta}, y_{\zeta}\right)=E_{n}\right)}{Q_{x} \times Q_{y}}
\end{gathered}
$$

式中, $H_{\mathrm{m}}\left(E_{m}, E_{n}\right)$ 为均值邻域二维直方图。将场图 谱相似度值定义为不同场图谱在各场强点处均值二 维直方图最小值之和。将大于相似度阈值的场图谱 
聚类为相同图谱, 得到车路场空间态势数据库 $D_{s}$

$$
D_{s}=\left\{D_{s, i}=\Omega_{j}, i=1, \cdots, w, j=1,2, \cdots, u\right\}
$$

式中, $w$ 为车路场空间态势数目, $u$ 为特定类型车 路场空间态势对应的场图谱数目。

\subsection{2 车路空间拓扑结构提取}

认为典型车路场空间态势下的主车空间位置服 从特定概率分布 $P\left(S_{t}=\left(S_{t, x}, S_{t, y}\right)\right)$, 得到车路场空间态 势数据库对应的主车空间位置集合 $\xi$, 如式(16)所示

$$
\xi=\left\{\xi_{i}=\left(x_{s}, y_{s}\right) \sim P\left(x_{s}, y_{s}\right), i=1,2, \cdots, w\right\}
$$

式中, $S_{t, x}$ 和 $S_{t, y}$ 为特定车路场空间态势 $D_{s, i}$ 对应的 主车空问位置纵坐标及横坐标数目。 $\xi$ 为完全可观 集合, 在大规模自然驾驶场景中具有高密度、片段 式和强随机特性。故采用带有拓扑学习特性的生长型 神经气(Growing neutral gas, GNG)算法获得特定车路 场空间态势中车辆状态连续拓扑结构。该算法为无监 督增量式拓扑学习算法 ${ }^{[20]}$, 其以增量式方式创建车 路场空间态势中的车辆状态拓扑图, 如式(17)所示

$$
G_{N}=\{\chi, \varepsilon\}
$$

式中, $\chi$ 和 $\varepsilon$ 分别表示车辆状态拓扑图的节点集和边 缘集。每个节点 $\left\{\chi^{[i]}, i=1,2, \cdots, G\right\}$ 及其对应的 特征向量 $\lambda^{[i]}=\left[\chi^{[i]} . S_{t, x}, \chi^{[i]} . S_{t, y}\right]^{T} \in R^{g}$ 构成 GNG 算法 样本空间的一个子流形。边缘集 $\varepsilon$ 为 $G N G$ 节点间的 连接关系, 用来建立样本空间的拓扑结构, 下式表 示节点 $\chi^{[i]}$ 及节点 $\chi^{[j]}$ 为拓扑相连关系

$$
e^{[i, j]}=\left[\chi^{[i]}, \chi^{[j]}\right] \in \varepsilon, i, j \in\{1,2, \cdots, G\}
$$

将主车空间位置集合 $\xi$ 作为算法输入, 节点集 $\chi$ 的学习过程为寻找最小期望偏差过程, 如下式

$$
\chi^{*}=\arg \min \chi \sum_{i=1}^{G} \int D_{e}\left(\left(x_{s}, y_{s}\right), \lambda^{[i]}\right) P\left(S_{\mathrm{t}}\right) \mathrm{d} x
$$

式中, $D_{e}$ 为欧式距离度量, 如式(20)所示

$$
D_{e}\left(S_{t}, \lambda^{[i]}\right)=\left\|S_{t}, \lambda^{[i]}\right\|_{2}
$$

\subsection{3 车辆运动模式生成}

认为典型车路场空间态势下主车纵侧向速度服 从特定运动模式, 根据其时变及统计特性, 选择可 表时空变化特征的高斯过程回归(Gaussian process regression, GPR)进行泛化。高斯过程是任何服从高 斯联合分布的有限随机变量集, 如式(21)所示

$$
y=f(X)+l, f \sim \mathrm{GP}(\eta, \vartheta), l \sim \mathrm{N}\left(0, \sigma_{1}^{2}\right)
$$

式中, $f(X)$ 为服从高斯过程的带有均值 $\eta(X)$ 和协方差 $\vartheta\left(X, X^{\prime}\right)$ 的函数。对于特定训练集 $\{X, y\}, X$ *映射 的输出变量 $y^{*}$ 后验概率分布服从下式正态分布

$$
y^{*} \sim \mathrm{N}\left(\bar{y}^{*}, \Sigma_{y^{*}}\right)
$$

$$
\begin{aligned}
& \bar{y}^{*}=\eta\left(X^{*}\right)+\vartheta^{T}\left(X, X^{*}\right) \vartheta^{-1}(X, X)(y-\eta(X)) \\
& \Sigma_{y^{*}}=\vartheta\left(X^{*}, X^{*}\right)-\vartheta^{T}\left(X, X^{*}\right) \vartheta^{-1}(X, X) \vartheta\left(X, X^{*}\right)
\end{aligned}
$$

车辆运动模式生成过程即为搜索车辆空间位置 与该空间处车辆速度映射关系的过程, 运动模式映 射关系可表示为 $M_{P}:\left(S_{t, x}, S_{t, y}\right)^{\mathrm{T}} \rightarrow\left(v_{t, x}, v_{t, y}\right)^{\mathrm{T}}$ 。当给 定车路空间拓扑结构后, 车辆运动模式如式(23)所示

$$
v_{M} \sim M_{p}(x)=N\left(\bar{v}_{M}, \Sigma_{v_{M}}\right) \quad \forall S_{t}^{\mathrm{T}} \in \xi
$$

GPR 训练过程的输入为车辆空间位置与该 空间处车辆纵侧向速度, 回归过程的输入为车辆 的空问位置, 输出为该空问处车速。采用零均值 函数 $\eta(X)$ 和平方指数协方差函数 $\vartheta\left(X, X^{\prime}\right)$ 。模型 的超参数通过最大化训练数据中观测值的似然 函数值学习得到。

\subsection{4 车路时空状态耦合}

由于实车数据均为带有传感噪声的片段式 数据, 根据车路空间拓扑结构及车辆运动模式, 将虚拟随机车路场模型 $H_{r}$ 抽象为由互斥区域 $R_{e}$ 、 各 $R_{e}$ 内车路拓扑结构 $\left\{\chi_{R}, \varepsilon_{R}\right\}$ 、不同 $R_{e}$ 间车辆运 动模式 $M_{R}$ 及不同 $R_{e}$ 间状态转移矩阵 $\Lambda$ 组成的概 率化模型, 下角标 $\mathrm{r}$ 为交通规则, 故 $H_{\mathrm{r}}$ 及各变量 如式(24)所示

$$
\left\{\begin{array}{l}
H_{r}=\left\{R_{e},\left(\chi_{R}, \varepsilon_{R}\right), M_{R}, \Lambda\right\} \\
R_{e}=\left\{R_{e}^{i}, i=1,2, \cdots, \varpi, R_{e}^{i} \cap R_{e}^{j}=\varphi, \forall i \neq j\right\} \\
\Lambda=P\left(R_{e}^{i} \mid R_{e}^{j}, r\right)
\end{array}\right.
$$

在给定 $R_{e} 、\left\{\chi_{R}, \varepsilon_{R}\right\}$ 和 $M_{R}$ 时, $\Lambda$ 为车路时空状 态耦合的关键, $\Lambda$ 的推导如下式

$$
\left\{\begin{array}{l}
P\left(\Theta_{t+\Delta t} \mid U^{[i]}\right)=\prod_{j=1}^{\sigma}\left(u_{R}^{[i]}\right)^{\psi_{i+\Delta t}^{[i]}} \\
\boldsymbol{U}^{[i]}=\left[u_{R, 1}^{[i]} \cdots u_{R, \sigma}^{[i]}\right] \quad \sum_{j=1}^{\sigma} u_{R, j}^{[i]}=1
\end{array}\right.
$$

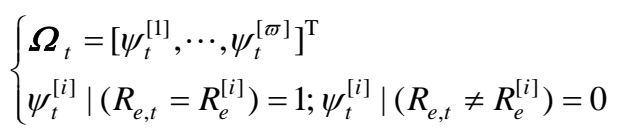

式中, $\boldsymbol{U}^{[i]}$ 为 $\Lambda$ 对应 $R_{e}{ }^{i}$ 的状态转移行量, $\Omega_{t}$ 表示 $t$ 时刻 $R_{e, t}$ 的 $\varpi$ 个互斥状态。式(25)为典型的多项式概 率分布, 故可以采用观测集 $O_{\Omega}=\left\{o_{1}, o_{2}, \cdots, o_{M}\right\}$ 的最大似然函数进行求解, 下角标 $M$ 表示 $O_{\Omega}$ 由 $M$ 个独立的观测矢量组成, 每个观测矢量 $o_{i}$ 均定义为 $o_{i}=\left\{\psi_{t+\Delta t}^{[1]}, \psi_{t+\Delta t}^{[2]} \cdots, \psi_{t+\Delta t}^{[\pi]}\right\}=\Omega_{t+\Delta t}$, 故 $O_{\Omega}$ 的最大似然 函数如式(27)所示

$$
\left(\boldsymbol{U}^{[i]}\right)^{M}=\underset{U^{[i]}}{\arg \max } \ln \mathrm{P}\left(O_{\Omega} \mid \boldsymbol{U}^{[i]}\right)
$$

车辆在 $t+\Delta t$ 时刻空问概率分布 $P\left(S_{t+\Delta t} \mid R_{e, t}=\mathrm{R}_{e}{ }^{[\mathrm{i}]}\right)$ 如式(28)所示 


$$
\begin{gathered}
P\left(S_{t+\Delta t} \mid R_{e, t}=R_{e}^{[i]}\right)= \\
\sum_{S_{t}} P\left(S_{t+\Delta t} \mid S_{t}, R_{e}^{[i]}\right) P\left(S_{t} \mid R_{e}^{[i]}\right)= \\
\sum_{S_{t}} \sum_{v_{M}} \underbrace{P\left(S_{t+\Delta t} \mid S_{t}, v_{\mathrm{M}}\right)}_{\text {速度积分 }} \underbrace{P\left(v_{M} \mid S_{t}\right)}_{G R P} \underbrace{P\left(S_{t} \mid R_{e}^{[i]}\right)}_{\text {观测值 }}
\end{gathered}
$$

式中, 观测值函数取值为布尔量且仅在 $S_{t} \in R_{\mathrm{e}}^{[i]}$ 时取 1 , 速度积分部分按 $S_{t+\Delta t}=S_{t}+v_{t} \cdot \Delta_{t}$ 计算, 故可通过预测车辆 空间分布规律估算 $O_{\Omega}$, 最大似然函数如式(29)所示

$$
u_{R, j}^{[i]}=M_{j} / M \quad \forall j \in\{1, \cdots, \varpi\}
$$

式中, $M_{j}$ 为使 $\psi_{t+\Delta t}^{[j]=1}$ 的观测值数目。

\section{3 驾驶能力机理分析}

\subsection{1 纵侧向驾驶能力离线辨识模型}

鉴于微观 RVRF 的动态强随机粒子特性及驾驶 人驾驶能力的时变、高阶非线性及动态特性, 选择 Hammerstein 辨识过程作为 RVRF 激励下的纵侧向 驾驶能力辨识模型, 如图 6 所示。Hammerstein 辨 识过程由静态非线性环节和动态线性环节串联而成。 在微观 RVRF 激励下, 本车状态及其与其他交通参 与物的相对状态组成模型输入 $S_{t}(k)$, 驾驶能力辨识 模型根据模型输入及模型自身属性, 输出踏板信号或 转向盘转角信号, 故纵侧向驾驶能力辨识模型均为 MISO 系统, 通过转向盘转角阈值进行逻辑切换。

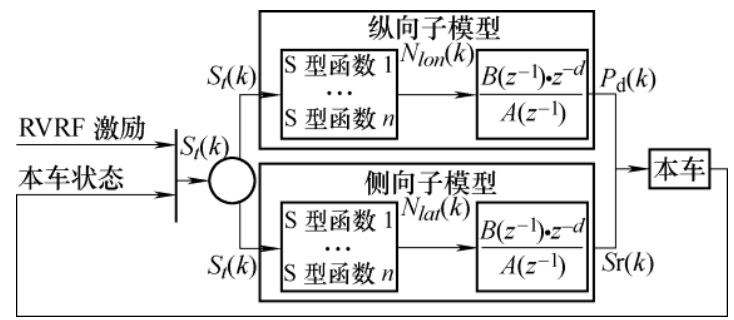

图 6 基于 Hammerstein 辨识过程的驾驶能力辨识模型

辨识模型中的静态非线性环节可以选用诸如死 区函数、 $\mathrm{S}$ 型函数或饱和函数等多种函数。辨识模 型中的动态线性环节可以表示为

$$
\begin{aligned}
& A\left(z^{-1}\right) \cdot O_{p}(k)=B\left(z^{-1}\right) \cdot z^{-d} \cdot N(k) \\
& \left\{\begin{array}{l}
A\left(z^{-1}\right)=1+a_{1} \cdot z^{-1}+\cdots+a_{q} \cdot z^{-q} \\
B\left(z^{-1}\right)=b_{1} \cdot z^{-1}+\cdots+b_{n} \cdot z^{-n}
\end{array}\right.
\end{aligned}
$$

式中, $O_{p}(k)$ 为制动及油门踏板合成的踏板信号 $P_{\mathrm{d}}(k)$ 与转向盘转角信号 $S_{\mathrm{r}}(k)$ 的集合, $N(k)$ 为纵侧向驾驶 能力辨识模型中静态非线性环节的输出组成的集合 $\left\{N_{\mathrm{n}}(k), N_{\mathrm{a}}(k)\right\}, q$ 和 $n$ 为动态线性环节的阶次, $d$ 为输入延迟阶次且定义为采样时间的整数倍。

\subsection{2 驾驶能力离线辨识模型参数解耦与降维}

经过大量数据训练后的驾驶能力辨识模型中, 静态非线性环节和动态线性环节所包含的模型参数
是表征驾驶能力内在属性的关键数据, 故将其作为 驾驶能力评价的数据样本。为避免数据样本彼此信 息重叠，同时保证在表达相同模型特征前提下，尽 可能降低数据样本空间维度，采用主成分分析法 (Principal component analysis, PCA) ${ }^{[21]}$ 对辨识模型的 关键参数进行解耦和降维。设集合 $H=\left\{H_{\mathrm{o}}, H_{\mathrm{a}}\right\}$ 表示 驾驶能力辨识模型参数维度, 其中 $H_{\mathrm{o}}$ 和 $H_{\mathrm{a}}$ 分别表 示纵侧向模型参数个数。设集合 $E=\left\{E_{0}, E_{\mathrm{a}}\right\}$ 表示驾 驶能力各循环试验中单次测试的数目, 其中 $E_{\mathrm{o}}$ 和 $E_{\mathrm{a}}$ 分别表示微观 RVRF 激励下的循环试验数目。可得 到 $H$ 维模型参数对应的 $E$ 个观测变量的数据集 $\boldsymbol{X}$ 为

$$
\boldsymbol{X}=\left(\begin{array}{cccc}
x_{11} & x_{12} & \cdots & x_{1 E} \\
x_{21} & x_{22} & \cdots & x_{2 E} \\
\vdots & \vdots & \vdots & \vdots \\
x_{H 1} & x_{H 2} & \cdots & x_{H E}
\end{array}\right)=\left(x_{1}, x_{2}, \cdots, x_{E}\right)
$$

基于驾驶能力辨识模型参数数据集 $\boldsymbol{X}$ 的 PCA 算法步骤如图 7 所示。
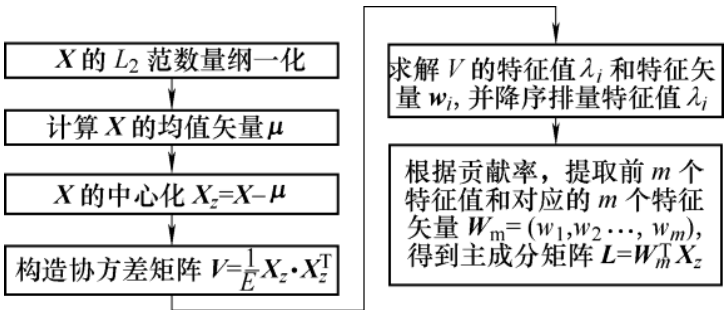

图 7 主成分分析算法步骤

将前 $m$ 个主成分特征值之和与全部特征值之和 所占百分比定义为主成分贡献率, 驾驶能力辨识模 型的独立参数维度由主成分累积贡献率 $M_{\mathrm{m}}$ 确定, 如式(33)所示

$$
M_{m}=\sum_{i=1}^{m} \lambda_{i} / \sum_{i=1}^{E} \lambda_{i}
$$

一般取 $M_{m} \geqslant 85 \%$ 对应的 $m$ 值作为主成分个数, 最终可得到 $m \times E$ 的主成分矩阵 $\boldsymbol{L}$, 如式(34)所示

$$
\boldsymbol{L}=\left(\begin{array}{cccc}
l_{11} & l_{12} & \cdots & l_{1 E} \\
l_{21} & l_{22} & \cdots & l_{2 E} \\
\vdots & \vdots & \vdots & \vdots \\
l_{m 1} & l_{m 2} & \cdots & l_{m E}
\end{array}\right)=\left(l_{1}, l_{2}, \cdots, l_{E}\right)
$$

\subsection{3 驾驶能力分类方法}

典型驾驶能力分类结果是分类驾驶能力数据库 及实时辨识驾驶能力的基础, 为保证驾驶能力分类 结果具有较高的置信度，采用主客观相结合的方式 对驾驶能力进行分类, 驾驶能力辨识模型的模型参 数样本属于驾驶能力分类模型集合

$$
D_{c}=\{\text { 强, 较强, 中, 较弱, 弱 }\}
$$


设计驾驶能力主观问卷进行驾驶能力测试, 如 表 2 所示。问卷的第 1 题项为自填式量表问卷, 驾 驶人对自身特性进行主观性评估 ${ }^{[22]}$, 在每次驾驶能 力数据采集试验结束后在不受干扰的情况下自主填 写, 量表的第 2 题项为他人评价驾驶人部分, 量表 各部分的评估方式均按照集合 $D_{c}$ 中元素划分, 并对 应相应分数。驾驶人完成循环试验各单次测试后, 均可得问卷题项 1 和题项 2 问卷结果, 取各次循环 试验中两题项的相一致结果作为问卷的有效结果。

表 2 驾驶能力问卷题项

\begin{tabular}{lccccc}
\hline \multicolumn{1}{c}{ 题项内容 } & 1 分 & 2 分 & 3 分 & 4 分 & 5 分 \\
\hline 自身驾驶能力评估 & 弱 & 较弱 & 中 & 较强 & 强 \\
驾驶人驾驶能力评估 & 弱 & 较弱 & 中 & 较强 & 强 \\
\hline
\end{tabular}

驾驶能力分类模型主要包含粒子群聚类过程及 聚类中心与驾驶能力类型映射部分。首先采用粒子 群算法对驾驶能力进行聚类。粒子群聚类算法是一 种有效的全局寻优算法, 在进化过程中同时记忆位 置和速度信息, 通过跟随当前最优解完成全局搜索 更新, 使粒子可以很快收玫于最优解。用于粒子群 群搜索的数据集 $\boldsymbol{L}$ 具有 $m$ 个独立参数特征及对应的 $E$ 个观测变量, 基于粒子群聚类的驾驶能力分类即 为在 $\boldsymbol{L}$ 中找到可以使可以使类内离散度和达到最小 的划分, 如式(36)所示

$$
J=\sum_{j=1}^{G} \sum_{X_{q} \in \omega_{j}} d\left(\boldsymbol{L}_{q}, \boldsymbol{L}^{\boldsymbol{\omega}_{j}}\right)
$$

式中, $\boldsymbol{L}^{\omega_{j}}$ 为第 $j$ 个聚类中心, $G$ 为聚类数目, $d\left(\boldsymbol{L}_{\mathrm{q}}\right.$, $\boldsymbol{L}^{\omega_{j}}$ )为样本到对应聚类中心距离。每个粒子均为粒子 群的一个可行解且均由粒子位置、粒子速度和粒子 适应度等三部分组成, 如式(37)所示

$$
P_{s, i}=\left\{L_{o, i}, V_{e, i}, F_{i}\right\} \quad i=1,2, \cdots, H
$$

式中, $P_{s, i}$ 为第 $i$ 个粒子, $H$ 为粒子数目。 $L_{\mathrm{o}}$ 和 $V_{\mathrm{e}}$ 分别为粒子位置和速度。 $F$ 为粒子适应度, 可通过 $F=k / J$ 计算得到, 其中 $k$ 为常数。每个粒子在进化过 程中记忆包含其位置和适应度的个体最优解 $p_{i, d}$, 整 个粒子群记忆其经历的最优位置和适应度的全局最 优解 $p_{g, d}$ 。粒子群通过更新方程得到其速度和位置, 如式(38)、(39)所示

$$
\begin{gathered}
v_{i, d}(t+1)=\delta \cdot V_{e, i, d}(t)+\eta_{1} \cdot \operatorname{rand}() \cdot\left(p_{i}-L_{o, i d}(t)\right)+\cdots \\
\eta_{2} \cdot \operatorname{rand}() \cdot\left(p_{g}-L_{o, i, d}(t)\right) \\
L_{o, i d}(t+1)^{\prime}=L_{o, i d}(t)+V_{e, i d}(t+1)^{\prime}
\end{gathered}
$$

式中, $V_{e, i, d}(t+1)$ 为在第 $t+1$ 次迭代中第 $d$ 维的第 $i$ 个粒子速度, $\delta$ 是惯性权重系数, $\eta_{1}$ 和 $\eta_{2}$ 为加速系 数, $\operatorname{rand}()$ 是范围为 $(0,1)$ 的随机数。 $V_{\mathrm{e}, \max }$ 和 $V_{\mathrm{e}, \min }$
为 $V_{e, i, d}(t+1)$ 的上下限

$$
\begin{cases}V_{e, i, d}(t+1)=v_{\max } & V_{e, i, d}(t+1)>V_{e, \text { max }} \\ V_{e, i, d}(t+1)=v_{\min } & V_{e, i, d}(t+1)<V_{e, \text { min }}\end{cases}
$$

由于聚类后的聚类结果尚不具有物理意义, 故 需要建立聚类结果与集合 $D_{c}$ 中各元素的映射关系。 基于粒子群聚类的聚类结果 $S_{c}$ 和基于量表分析的分 类结果 $O_{c}$ 如式(41)、(42)所示

$$
\begin{aligned}
& S_{c}=\left\{S_{c, i, j}, i=1, \cdots, 5, \sum_{i}^{5} j=E\right\} \\
& O_{c}=\left\{O_{c, i, u}, i=1, \cdots, 5, \sum_{i}^{5} u=E\right\}
\end{aligned}
$$

与集合 $S_{c}$ 中特定元素交集最大的集合 $O_{c}$ 中元素 即为相同类型驾驶能力元素, 元素交集包含的单次测 试为有效值, 其余为无效值, 如式(43)、(44)所示

$$
\begin{aligned}
& S_{o}=\left\{S_{o, r, y}, r=1,2, \cdots, 5, \sum_{i}^{5} y=e \leqslant E Z\right\} \\
& S_{o, r, y}=\left\{O_{c, r, u} \cap S_{c, i, j}, \max y, r=1,2, \cdots, 5\right\}
\end{aligned}
$$

\subsection{4 基于混合高斯模型的驾驶能力实时辨识}

在完成驾驶能力机理分析和分类出典型驾驶能 力数据库的基础上, 采用混合高斯隐马尔科夫模型 (Gaussian multi-dimension hidden markov model, GM-HMM), 在微观 RVRF 激励下实时辨识驾驶能 力。微观 RVRF 激励下, 驾驶人的操作信号、主车 状态以及交通参与物与主车的相对状态信号均为连 续的时间序列且影响下一采样时刻的状态，可以将 该物理过程抽象为隐马尔科夫过程。集合 $D_{\mathrm{c}}$ 中每个 元素可表示为与之对应的驾驶能力实时辨识过程 (Driving capability real time identification process, DCRTP)。由于采集到的驾驶人观测变量均为连续时 问序列, 故可以采用多维高斯模型表示观测变量的 概率密度函数 ${ }^{[23]}$ 。因此, 可将每个 DCRTP 模型映 射为一个 GM-HMM。GM-HMM 包含一组隐状态 $q_{t}$ 和及每一状态下 $\kappa$ 组可能的观察值输出 ${ }^{[24]}$ 。DCRTP 模型精度随 GM-HMM 阶数的增加而提高, 但阶数 过高会大大降低计算速度。考虑到主车与交通车辆 运动模式的时间序列, 其下一时刻的运动状态主要 受到上一时刻运动状态的影响, 故 DCRTP 子模型 采用从左至右结构的一阶马尔科夫过程。初始状态 分布定义为

$$
\begin{gathered}
\pi=\left\{\pi_{i}\right\} \\
\pi_{i}=P\left[q_{1}=i\right] \quad 1 \leqslant i \leqslant N
\end{gathered}
$$

状态转移概率矩阵定义为 


$$
\begin{gathered}
\boldsymbol{T}_{r}=\left\{a_{i, j}\right\} \\
a_{i, j}=P\left[q_{t+1}=j \mid q_{t}=i\right] \quad 1 \leqslant i, j \leqslant N
\end{gathered}
$$

式中, $q_{t}$ 表示 $t$ 时刻的状态。 $\boldsymbol{O}$ 为一组可观测序列 集合, $\boldsymbol{O}=\left\{V_{1}, V_{2}, \cdots, V_{\kappa}\right\}, V$ 代表可能的观察值, 下角标 $\kappa$ 为每一状态对应的可能观察值数目。设状 态 $j$ 的观察概率矩阵为 $\boldsymbol{T}_{\mathrm{B}}=\left\{b_{j}(\boldsymbol{O})\right\}$, 由于在连续观 测序列中 $\kappa \rightarrow+\infty$, 故 $\boldsymbol{T}_{\mathrm{B}}=\left\{b_{j}(\boldsymbol{O})\right\}$ 为状态 $j$ 输出的观 察值概率密度函数, 表达式如式(49)所示

$$
b_{j}(\boldsymbol{O})=\sum_{k=1}^{M} c_{j, k} N\left(\boldsymbol{O} \mid \mu_{j, k}, \Sigma_{j, k}\right) \quad 1 \leqslant j \leqslant N
$$

式中, $c_{j, k}$ 为第 $j$ 个状态第 $k$ 次混合的混合权重系数, $M$ 为高斯混合数, $N\left(\boldsymbol{O} \mid \mu_{j, k}, \sum_{j, k}\right)$ 是均值为 $\mu$ 、协方 差为 $\sum$ 的高斯分布概率密度函数。权重系数 $c_{j, k}$ 为

$$
\begin{gathered}
\sum_{k=1}^{M} c_{j, k}=1 \quad c_{j, k} \geqslant 0 \quad 1 \leqslant j \leqslant N \quad 1 \leqslant k \leqslant M \\
\int_{-\infty}^{+\infty} b_{j}(\boldsymbol{O}) \mathrm{d} \boldsymbol{O}=1 \quad 1 \leqslant j \leqslant N
\end{gathered}
$$

故 DSIM 可由 $N$ 个隐状态通过一个元组 $\lambda$ 定义

$$
\begin{gathered}
\lambda=\left(\pi, T_{r}, c, \mu, \Sigma\right) \\
\log \operatorname{lik}(\theta)=\ln [P(\boldsymbol{O} \mid \lambda)]
\end{gathered}
$$

\section{4 虚拟 RVRF 中驾驶能力分析结果}

\section{1 驾驶能力机理分析逻辑框架}

\subsection{1 车路场空间态势聚类结果分析}

以直路类三车道无侧方停车且主车在中间车道 行驶的自然交通场景采集的对应数据为例, 得到两 个特定时刻下对应的场图谱如图 8 所示。该场图谱
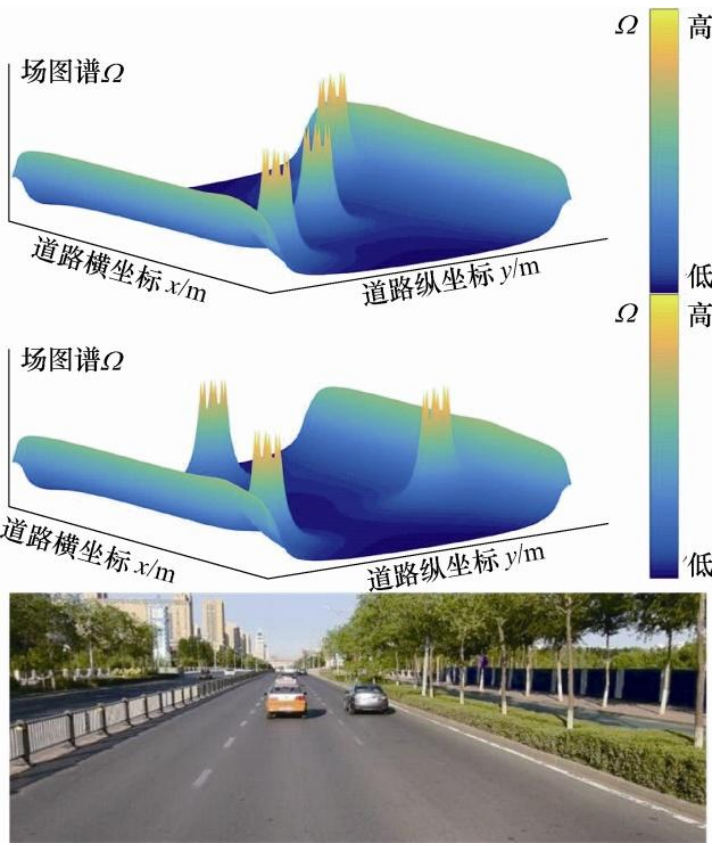

图 8 直路类自然交通场景时间切片下的场图谱表示
由三辆交通车的 $E_{k}$ 、路沿石及护栏的 $E_{p}$ 及三辆交通 车的 $E_{i}$ 组成。

通过两个特定时刻下对应的场图谱计算得到相 应的均值邻域二维直方图如图 9 所示，对应的相似 度直方图结果如图 10 所示，相似度为 $79.163 \%$ 。将 相似度大于 $95 \%$ 的场图谱对应的自然交通场景数据 的时间切片划分为同一类型，车路场空间态势聚类

\begin{tabular}{|c|c|c|c|}
\hline 工况类型 & 一级子工况 & 二级子工况 & 聚类结果(类) \\
\hline 直路 & 单/双/三/多车道 & \multirow{5}{*}{$\begin{array}{l}\text { (1) 有无道路 } \\
\text { 侧方停车; } \\
\text { (2) 周围交通 } \\
\text { 车密度稀疏/正常/ } \\
\text { 稠密 }\end{array}$} & 182 \\
\hline 弯路 & $\begin{array}{l}\text { (1) 小/中/大弯路 } \\
\text { (2)单至多车道 }\end{array}$ & & 519 \\
\hline $\mathrm{T}$ 形路 & $\begin{array}{l}\text { (1)直角/非直角 } \\
\text { (2)单至三车道 }\end{array}$ & & 198 \\
\hline 交叉口 & 有/无交通灯 & & 341 \\
\hline 环岛 & $\begin{array}{l}\text { (1)大环岛/小环岛 } \\
\text { (2)进/出/绕行环岛 }\end{array}$ & & 447 \\
\hline
\end{tabular}
结果如表 3 所示。

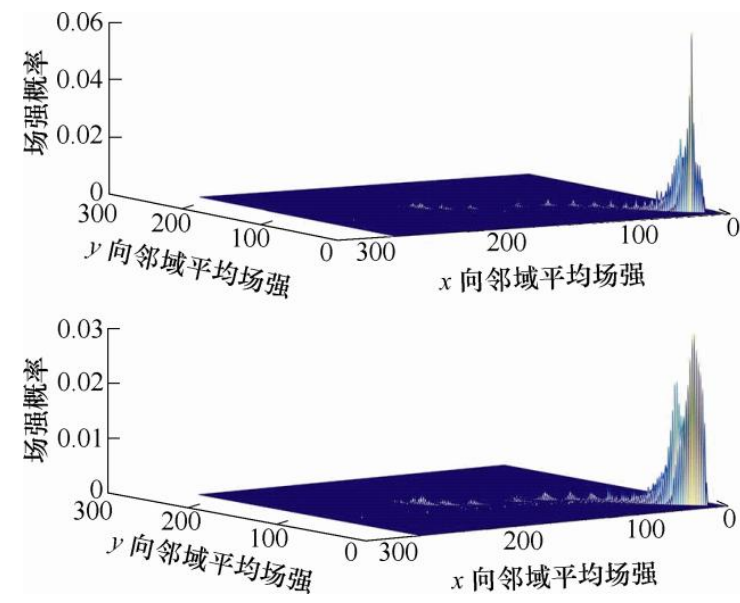

图 9 场图谱的均值邻域二维直方图计算结果

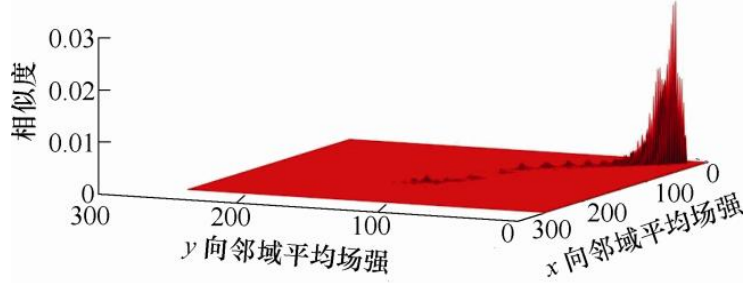

图 10 相似度直方图计算结果

表 3 车路场空间态势聚类结果

4.1.2 车路空间拓扑结构提取结果分析

直路类三车道无侧方停车场景聚类后的典型车 路场图谱及其对应的主车位置分布如图 11 所示。由 图可知, 在特定场图谱中, 主车按环绕低场强的方 式入场，具有强粒子性和随机性。

车路空间拓扑结构提取结果如图 12 所示。GNG 算法的均方根误差变化曲线如图 13 所示。 


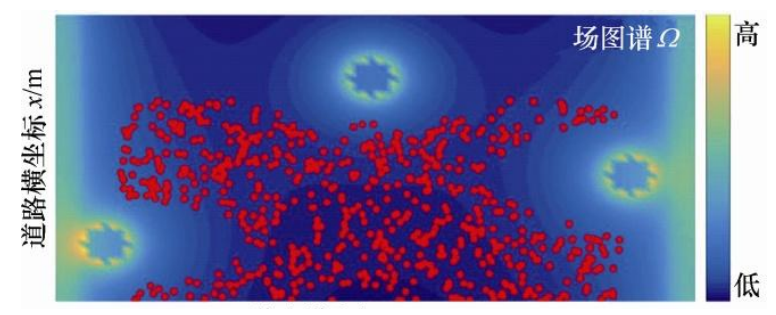

图 11 车路空间拓扑结构提取结果

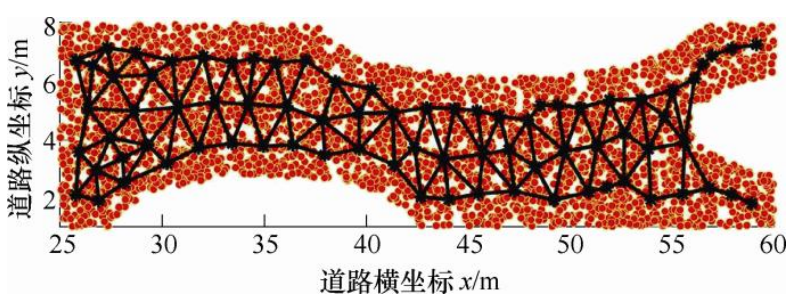

图 12 车路空间拓扑结构提取结果

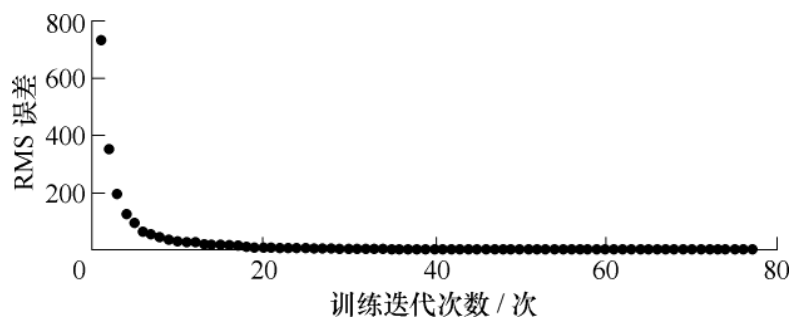

图 13 GNG 算法 RMS 误差

\subsection{3 车辆运动模式生成结果分析}

采用 $4 / 5$ 数据作为训练数据, $1 / 5$ 数据作为测试 数据。直路类三车道无侧方停车场景对应的车辆运 动模式如图 14 所示。图中上三角表示特定车路场空 间态势下的交通车位置, 圆圈表示主车位置, 箭头 为基于 GPR 算法的主车速度预测结果。如图可知, 在特定车路场空问态势下主车运动服从车路场物理 约束, 同时具有强粒子性和随机性。

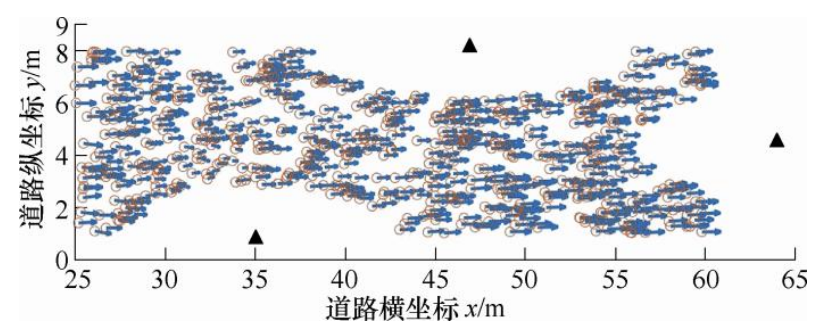

图 14 车辆运动模式速度流图

\subsection{4 虚拟 RVRF 车路时空状态耦合结果分析}

采用 Voronoi 多边形图法绘制的虚拟 RVRF 车 路时空状态耦合结果如图 15 所示。由图可知, 基于 GNG 算法的 $\left\{\chi_{R}, \varepsilon_{R}\right\}$ 为直路类三车道无侧方停车场 景对应场景中, Voronoi 多边形图法表示的互斥区域 $R_{e} 、 \mathrm{GPR}$ 算法得到的 $M_{R}$ 及不同 $R_{e}$ 问状态转移概率 共同构成 RVRF，主车在虚拟 RVRF 中下一时刻的 位置及运动状态由虚拟 RVRF 决定。

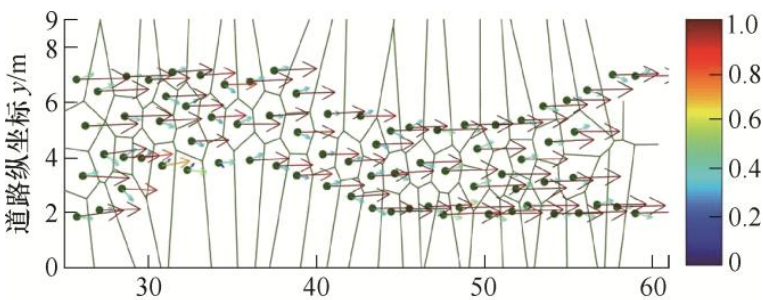

图 15 虚拟微观 RVRF 车路时空状态耦合结果

采用直路类三车道无侧方停车场景, 在 $\mathrm{NaSch}$ 模型中设定每个元胞长度为 $1.5 \mathrm{~m}$, 车辆长度为 $4.5 \mathrm{~m}$, 即每辆车占据 3 个连续元胞, 模型采样时问 设置为 $1 \mathrm{~s}$ 。虚拟 RVRF 设置交通车数量为 10 辆, 沿闭环场景循环行驶。虚拟宏观 RVRF 和经典 $\mathrm{NaSch}^{[25]}$ 宏观交通流波动结果对比如图 16 所示。如 图所示, 在多交通车辆交通流场景中, 虚拟宏观 RVRF 和经典 $\mathrm{NaSch}$ 在密度-流量波动上呈现高度一 致性, 且宏观交通流密度及流量均具有波动性。

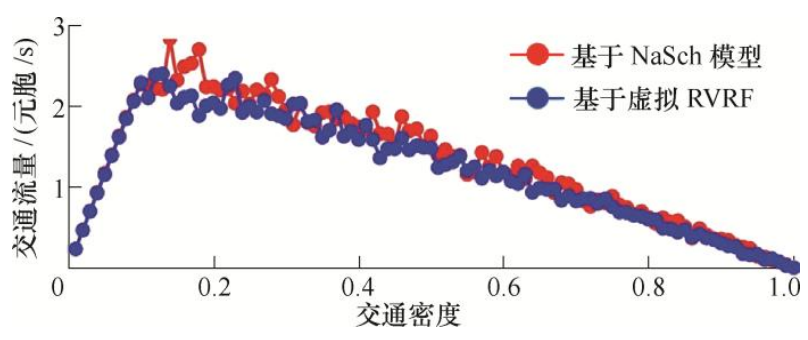

图 16 虚拟宏观 RVRF 交通流波动结果对比图

\section{2 虚拟 RVRF 中驾驶能力机理分析逻辑框架}

\subsection{1 驾驶能力辨识模型结果分析}

纵向子模型输入为本车急动度、本车加速度、 本车与前方交通车的相对速度和纵向相对距离, 输 出为加速踏板位置与制动踏板位置组成的踏板位置 信号; 侧向子模型的输入为本车横摆角速度、本车 侧向速度、与相邻前方交通车纵向相对速度及纵向 相对位移, 与相邻侧方交通车侧向相对位移及纵向 相对位移, 模型的输出为转向盘转角。各子模型的 静态非线性环节均选择 $\mathrm{S}$ 型函数, 动态线性环节阶 次 $q=3$ 和 $n=3$, 输入延迟阶次 $d=1$ 。编号 No. 1 驾驶 人的驾驶能力辨识模型结果如图 17 所示。图 17a 为驾驶人在直路类虚拟 RVRF 中第 1 次循环试验 No.165 单次测试的纵向驾驶能力辨识结果, 图 $17 \mathrm{~b}$ 为直路类虚拟 RVRF 中第 1 次循环试验 No.219 单次 测试的侧向驾驶能力辨识结果。由于驾驶能力具有 时变且渐变物理特性, 为验证纵向驾驶能力辨识模 型的有效性, 分别对驾驶人下一次出现纵侧向操纵 行为对应的试验编号, 即直路类虚拟 RVRF 中第 1 次循环试验 No.166 和 No.223 的驾驶能力数据进 行预测, 图 17c、17d 为预测结果。各被测驾驶人 


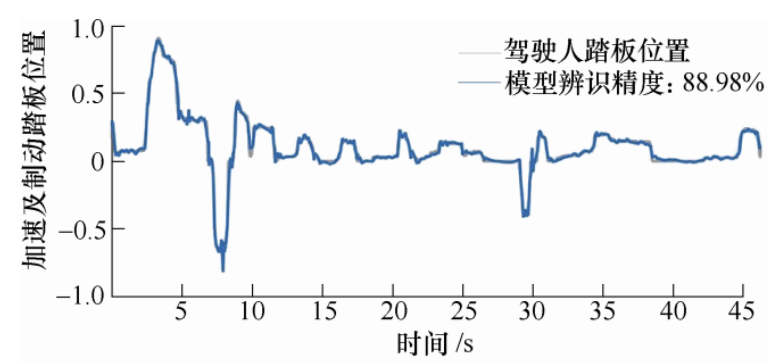

(a) 纵向子模型辨识结果

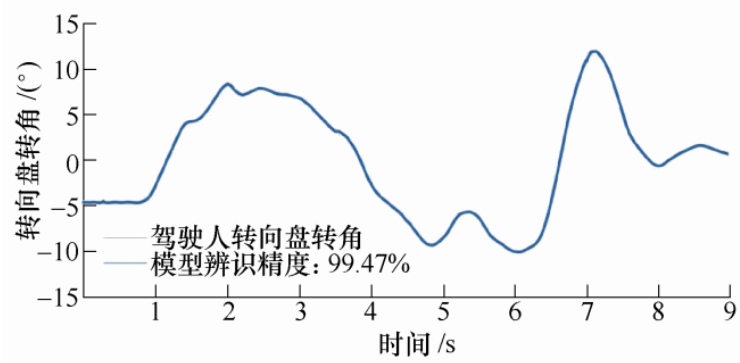

(b) 侧向子模型辨识结果

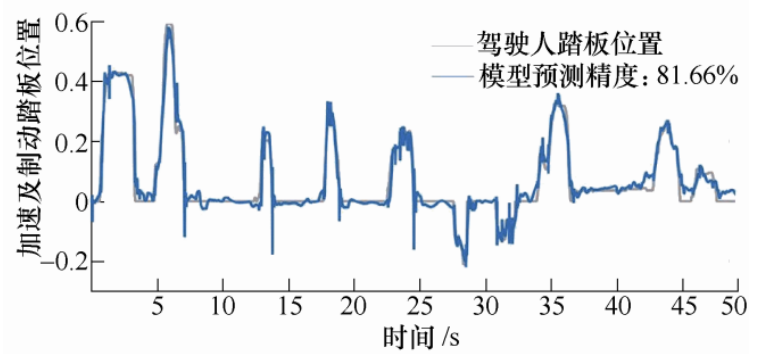

(c) 纵向子模型预测结果

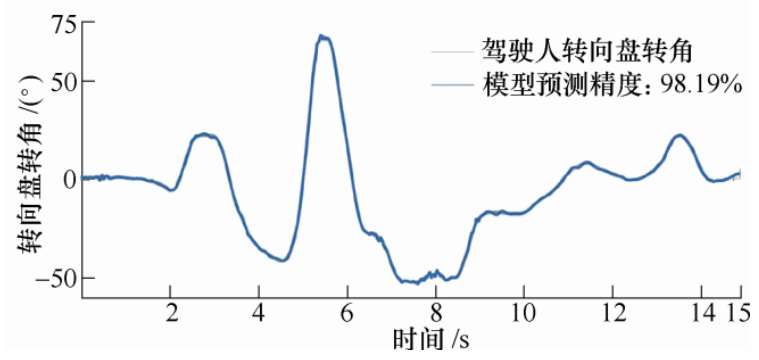

图 17 纵侧向驾驶能力辨识模型结果分析

的模型辨识及预测精度统计如表 4、5 所示, 由表可 知, 驾驶人纵侧向驾驶能力辨识子模型的平均辨识 及预测拟合精度均在 $85 \%$ 以上, 平均辨识拟合精度 分别为 $90.710 \%$ 和 $95.386 \%$, 平均预测拟合精度分 别为 $88.048 \%$ 和 $94.726 \%$ 。

表 4 纵向子模型辨识及预测精度统计

\begin{tabular}{ccc}
\hline 驾驶人编号 & 平均辨识拟合精度 $(\%)$ & 平均预测拟合精度 $(\%)$ \\
\hline 1 & 93.26 & 91.31 \\
2 & 91.47 & 88.44 \\
3 & 89.23 & 85.93 \\
4 & 90.37 & 87.62 \\
5 & 89.22 & 86.94 \\
\hline 总体均值 & 90.710 & 88.048 \\
\hline
\end{tabular}

表 5 侧向子模型辨识及预测精度统计

\begin{tabular}{ccc}
\hline 驾驶人编号 & 平均辨识拟合精度 $(\%)$ & 平均预测拟合精度 $(\%)$ \\
\hline 1 & 95.92 & 95.16 \\
2 & 96.77 & 95.93 \\
3 & 94.81 & 93.44 \\
4 & 96.42 & 96.21 \\
5 & 93.01 & 92.89 \\
\hline 总体均值 & 95.386 & 94.726 \\
\hline
\end{tabular}

综上可知, 基于 Hammerstein 过程的驾驶人纵侧向 驾驶能力辨识模型具有较高的辨识及预测拟合精度, 可用于辨识驾驶人的纵侧向驾驶能力。

\subsection{2 基于 PCA 的模型参数解耦与降维结果分析 \\ 驾驶能力辨识模型静态及动态环节 $H$, 如式(54)} 所示

$$
H_{\Phi}=\left\{S_{\Phi}, D_{\Phi}\right\} \quad \Phi=\left\{L_{\mathrm{o}}, L_{\mathrm{a}}\right\}
$$

式中, $S_{\Phi}$ 为各输入变量对应的静态非线性环节中 $S$ 型函数参数总维度, $D_{\Phi}$ 为动态线性环节参数维度, 各被测驾驶人 $H_{\Phi}$ 维度均相同, 如表 6 所示。编号 No.1 驾驶人的模型参数特征值和主成分累计贡献 率如表 7 所示。取 $M_{\mathrm{m}} \geqslant 85 \%$ 对应的 $m$ 值作为主成 分个数, 各被测驾驶人的纵侧向驾驶能力主成分维度 如表 8 所示。由表可知, 驾驶人的纵侧向驾驶能力主 成分维度均为其模型参数维度的 $1 / 3 \sim 1 / 4$ 。根据主成 分维度及对应特征向量, 进而可得主成分矩阵 $\boldsymbol{L}$ 。

表 6 驾驶能力辨识模型参数维度统计

\begin{tabular}{ccc}
\hline 题项内容 & $\Phi=L_{\mathrm{o}}$ & $\Phi=L_{\mathrm{a}}$ \\
\hline$S_{\Phi}$ & 29 & 48 \\
$D_{\Phi}$ & 20 & 30 \\
\hline 总计 & 49 & 78
\end{tabular}

表 7 面向模型参数的特征值和主成分累计贡献率

\begin{tabular}{ccc}
\hline 主成分编号 & 特征值(纵向/侧向) & 累计贡献率(纵向/侧向) \\
\hline 1 & $5.766 / 14.158$ & $12.436 / 17.848$ \\
2 & $5.014 / 8.444$ & $23.251 / 28.494$ \\
3 & $3.935 / 4.857$ & $31.737 / 34.617$ \\
4 & $3.554 / 3.766$ & $39.402 / 39.364$ \\
5 & $3.403 / 3.612$ & $46.741 / 43.918$ \\
6 & $3.125 / 3.476$ & $53.482 / 48.300$ \\
7 & $2.868 / 3.121$ & $59.667 / 52.235$ \\
8 & $2.382 / 2.689$ & $64.805 / 55.624$ \\
9 & $2.073 / 2.658$ & $69.276 / 58.976$ \\
10 & $1.902 / 2.529$ & $73.378 / 62.164$ \\
11 & $1.818 / 2.318$ & $77.299 / 65.087$ \\
12 & $1.681 / 2.260$ & $80.925 / 67.935$ \\
13 & $1.376 / 2.065$ & $83.894 / 70.539$ \\
14 & $1.111 / 1.848$ & $86.289 / 72.869$ \\
15 & $-/ 1.770$ & $-/ 75.100$ \\
16 & $-/ 1.680$ & $-/ 77.217$ \\
17 & $-/ 1.573$ & $-/ 79.200$ \\
18 & $-/ 1.402$ & $-/ 80.968$ \\
19 & $-/ 1.348$ & $-/ 82.667$ \\
20 & $-/ 1.217$ & $-/ 84.201$ \\
21 & $-/ 1.182$ & $-/ 85.691$ \\
\hline & &
\end{tabular}


表 8 各被测驾驶人驾驶能力主成分维度统计

\begin{tabular}{cc}
\hline 驾驶人编号 & 驾驶能力主成分维度(纵向/侧向) \\
\hline 1 & $14 / 21$ \\
2 & $16 / 24$ \\
3 & $14 / 22$ \\
4 & $16 / 23$ \\
5 & $15 / 22$ \\
\hline
\end{tabular}

4.2.3 基于主客观方法的驾驶能力分类结果分析

纵侧向驾驶能力主观问卷题项一致率定义为单 次循环试验中量表题项 1 和题项 2 得到的相同结果 对应的单次测试数与单次测试总数的百分比。编号 No.1 驾驶人在纵侧向各次循环试验中的主观问卷 题项一致率如表 9 所示。

表 9 典型纵侧向驾驶能力主观问卷题项一致率

\begin{tabular}{cc}
\hline 典型纵侧向驾驶能力主管问卷题项 & 一致率 $(\%)$ \\
\hline 纵向驾驶能力量表问卷 & $83.02,85.30,79.09,75.36,78.05$, \\
侧向驾驶能力量表问卷 & $76.40,82.82,74.33,82.61,74.95$ \\
& $91.07,93.90,90.03,94.35,95.83$, \\
\end{tabular}

将集合 $S_{c}$ 与 $O_{c}$ 中各元素交集的单次测试数与 集合 $O_{c}$ 中对应元素的单次测试数的比值定义为驾 驶能力主客观分类重合度, 重合度最大的主客观分 类类型所对应的单次测试结果, 即为单次循环试验 得到的有效分类结果。编号 No.1 驾驶人第 1 次纵向 循环试验包含 483 组单次测试, 第 1 次侧向循环试 验包含 673 组单次测试, 求得的纵侧向驾驶能力主 客观分类重合度及分类结果如表 10、11。

表 10 典型纵向驾驶能力主客观分类重合度

\begin{tabular}{cccccc}
\hline \multirow{2}{*}{ 量表问卷 } & \multicolumn{5}{c}{ 客观分类 $(\%)$} \\
\cline { 2 - 6 } & 1 & 2 & 3 & 4 & 5 \\
\hline 弱 & 4.35 & 2.17 & 80.43 & 8.70 & 4.35 \\
较弱 & 17.98 & 1.12 & 7.87 & 70.79 & 2.25 \\
中 & 70.97 & 3.23 & 3.23 & 4.30 & 18.28 \\
较强 & 4.90 & 25.59 & 2.94 & 3.92 & 67.65 \\
强 & 8.45 & 63.38 & 4.23 & 9.86 & 14.08 \\
\hline
\end{tabular}

结论: 弱 $\rightarrow 3$; 较弱 $\rightarrow 4$; 中 $\rightarrow 1$; 较强 $\rightarrow 5$; 强 $\rightarrow 2$ 。

表 11 典型侧向驾驶能力主客观分类重合度

\begin{tabular}{cccccc}
\hline \multirow{2}{*}{ 量表问卷 } & \multicolumn{5}{c}{ 客观分类 $(\%)$} \\
\cline { 2 - 6 } & 1 & 2 & 3 & 4 & 5 \\
\hline 弱 & 4.08 & 77.55 & 14.29 & 3.06 & 2.63 \\
较弱 & 14.89 & 2.13 & 73.05 & 6.38 & 3.55 \\
中 & 68.91 & 5.88 & 2.52 & 19.33 & 3.36 \\
较强 & 9.42 & 2.90 & 6.52 & 66.67 & 14.49 \\
强 & 4.81 & 1.92 & 5.77 & 11.54 & 75.96 \\
\hline 结论: 弱 $\rightarrow 2 ;$ & 较弱 $\rightarrow 3 ;$ 中 $\rightarrow 1 ;$ 较强 $\rightarrow 4 ;$ 强 $\rightarrow 5$ 。 \\
\hline
\end{tabular}

由表 10、11 分别计算出编号 No.1 驾驶人的第 1 次纵向及侧向循环试验包含单次测试有效值组数 分别为 280 组和 432 组。面向数据集 $L$ 的典型纵侧 向驾驶能力分类结果如图 18 所示。作为驾驶人驾驶 习性、驾驶技能及驾驶状态的综合表征, 在单次循 环试验中, 纵侧向驾驶能力具有非线性时变且渐变 物理特性, 在相邻若干次单次测试中, 驾驶能力还 具有波动性和随机性。

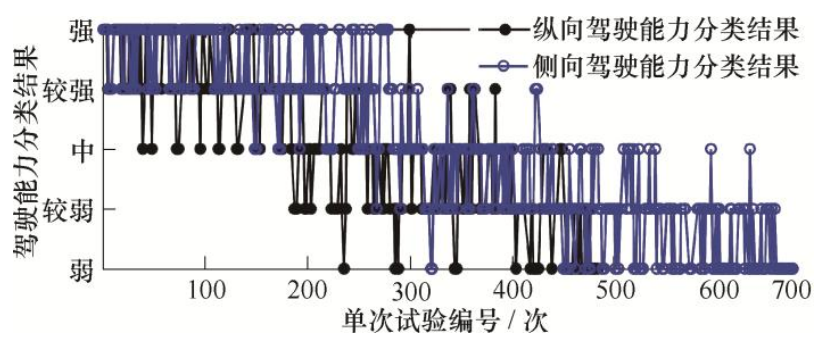

图 18 典型直路类虚拟 RVRF 中驾驶能力分类结果

\subsection{4 基于 GM-HMM 的驾驶能力实时辨识结果}

根据有效分类结果选择数据样本用于 DCRTP 的训练和辨识。用于训练辨识模型的样本数为采集 数据量的 $3 / 5$, 采用 Baum-Welch 方法完成各 DCRTP 训练。用于辨识的样本数为采集数据量的 $2 / 5$ 。将模 型输入信号输入至 5 个 DCRTP 中, 最大的模型似 然率对应的 DCRTP 即为所辨识驾驶人的驾驶能力 类型。编号 No.1 号驾驶人在直路类虚拟 RVRF 中第 1 次循环试验 No.200 单次测试的驾驶能力类型辨识 结果如表 12 所示。模型辨识过程中, 多维高斯混合 数 $M$ 、隐状态维数 $N$ 、模型训练时间长度 $T_{P}$ 以及辨 识时采用的模型辨识时间长度 $I_{P}$ 是影响辨识结果的 4 个主要因素, 采用正交试验方法对这 4 个因素进 行优化分析, 建立 $\mathrm{L}_{9}\left(3^{4}\right)$ 标准正交表, 如表 13 所示。 直路类虚拟 RVRF 中第 1 次循环试验下的正交试验 结果如表 14 所示, 表中的 $y_{p, v}, v=1,2,3$ 表示第 $p$ 因素 $v$ 水平对应的试验指标和; $\bar{y}_{p, v}, v=1,2,3$ 为 $y_{p, v}$ 的平均值, 每一因素中 $\bar{y}_{p, v}$ 的最大值对应的水 平为该组正交试验下该因素的最优水平, 各因素及 其最优水平的组合为正交试验的最优组合; $R_{p}$ 为第 $p$ 因素的极差, 按极差值大小即可判断各因素的主 次顺序, 其计算式如式(55)所示

$$
R_{p}=\max \left[\bar{y}_{p, 1}, \bar{y}_{p, 2}, \bar{y}_{p, 3}\right]-\min \left[\bar{y}_{p, 1}, \bar{y}_{p, 2}, \bar{y}_{p, 3}\right]
$$

表 12 No.1 驾驶人驾驶能力实时辨识结果

\begin{tabular}{ccc}
\hline DCRTP & 模型似然率 LogLik 值 & 辨识结果 \\
\hline 5 & $0.4162 \times 10^{5}$ & \\
4 & $0.2340 \times 10^{5}$ & \\
3 & $0.7251 \times 10^{4}$ & 5 \\
2 & $0.8016 \times 10^{4}$ & \\
1 & $0.2159 \times 10^{5}$ & \\
\hline
\end{tabular}


表 13 正交试验因素水平表

\begin{tabular}{ccccc}
\hline & \multicolumn{5}{c}{ 因素 } \\
\cline { 2 - 5 } 水平 & $\mathrm{A}(M)$ & $\mathrm{B}(N)$ & $\mathrm{C}\left(T_{\mathrm{P}}\right)$ & $\mathrm{D}\left(I_{\mathrm{P}}\right)$ \\
\hline 1 & 4 & 4 & 40 & 35 \\
2 & 8 & 5 & 50 & 45 \\
3 & 12 & 6 & 60 & 50 \\
\hline
\end{tabular}

表 14 正交试验辨识结果

\begin{tabular}{cccccc}
\hline 试验组号 & $\mathrm{A}$ & $\mathrm{B}$ & $\mathrm{C}$ & $\mathrm{D}$ & 准确率(\%) \\
\hline 1 & 1 & 1 & 1 & 1 & 86.3 \\
2 & 1 & 2 & 2 & 2 & 89.0 \\
3 & 1 & 3 & 3 & 3 & 90.6 \\
4 & 2 & 1 & 2 & 3 & 92.8 \\
5 & 2 & 2 & 3 & 1 & 94.7 \\
6 & 2 & 3 & 1 & 2 & 96.3 \\
7 & 3 & 1 & 3 & 2 & 95.2 \\
8 & 3 & 2 & 1 & 3 & 96.0 \\
9 & 3 & 3 & 2 & 1 & 96.9 \\
$y_{\mathrm{p}, 1}$ & 265.9 & 274.3 & 278.6 & 277.9 & \\
$y_{\mathrm{p}, 2}$ & 283.8 & 279.7 & 278.7 & 280.5 & \\
$y_{\mathrm{p}, 3}$ & 288.1 & 283.8 & 280.5 & 279.4 & \\
$\bar{y}_{\mathrm{p}, 1}$ & 88.63 & 91.43 & 92.87 & 92.63 & \\
$\bar{y}_{\mathrm{p}, 2}$ & 94.60 & 93.23 & 92.90 & 93.50 & $\sum_{i=1}^{3} y_{i}=837.8$ \\
$\bar{y}_{\mathrm{p}, 3}$ & 96.03 & 94.60 & 93.50 & 93.13 & \\
$\mathrm{Rp}$ & 7.40 & 3.17 & 0.63 & 0.50 & \\
优水平 & $\mathrm{A} 3$ & $\mathrm{~B} 3$ & $\mathrm{C} 3$ & $\mathrm{D} 2$ & \\
主次因素 & & $\mathrm{A}>\mathrm{B}>\mathrm{C}>\mathrm{D}$ & & \\
最优组合 & & $\mathrm{A} 3 \mathrm{~B} 3 \mathrm{C} 3 \mathrm{D} 2$ & & \\
\hline & & & & & \\
\hline & & &
\end{tabular}

编号 No.1 号驾驶人在直路类虚拟 RVRF 中第 2 次循环试验对应的驾驶能力在线辨识结果如图 19 所示。

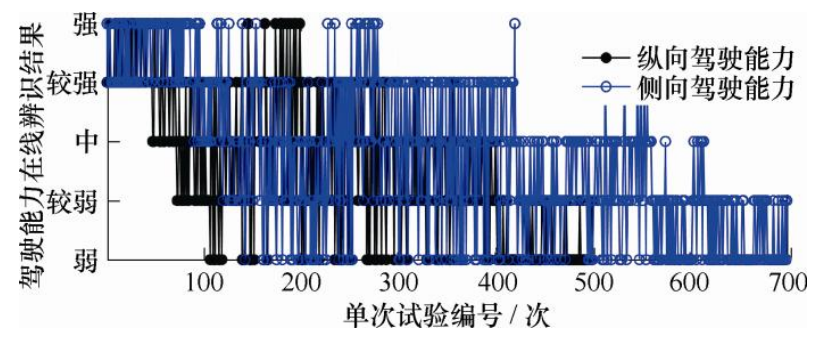

图 19 典型虚拟 RVRF 中驾驶能力在线辨识结果

\section{5 结论}

(1) RVRF 是虚拟仿真环境下车-车及车-路彼此 耦合形成的强随机稳态场, 是虚拟环境下对自然驾 驶场景中车-车及车-路作用关系的精细模型。通过
车路时空状态耦合建立的虚拟 RVRF, 在微观驾驶 场景中具有强随机粒子特性且在宏观驾驶场景中具 有波运动特性; 与经典 NaSch 模型具备相似波动性 和时空演化一致性, 虚拟 RVRF 可以客观反映交通 流的波粒二象性。

（2）驾驶能力是驾驶人随外界环境负荷变化而 渐变的对车辆把控的能力, 是个人驾驶习性、驾驶 技能及驾驶状态的综合体。在微观虚拟 RVRF 的驾驶 人在环智能仿真平台上采集数据; 基于 Hammerstein 模型建立纵侧向驾驶能力离线辨识模型，纵侧向子 辨识模型辨识精度分别为 $90.710 \%$ 和 $95.386 \%$, 预 测拟合精度为分别 $88.048 \%$ 和 $94.726 \%$ 。

(3) 纵侧向驾驶能力主成分解耦和降维后的 维度均为其模型参数维度的 $1 / 3 \sim 1 / 4$; 通过客观 粒子群聚类和主观量表分析相结合的驾驶能力分 类方式, 验证了驾驶能力呈现非线性时变且渐变 物理特性, 在若干次相邻重复试验中, 驾驶能力 呈现波动性和随机性; 通过 GM-HMM 实现驾驶 能力的实时辨识。通过对模型中关键参数进行正 交试验提高辨识准确率, 得到准确且可靠的驾驶 能力在线辨识结果。

\section{参 考 文 献}

[1] KAPLAN S, GUVENSAM M A, YAVUZ A G, et al. Driver behavior analysis for safe driving: A survey[J]. IEEE Transactions on Intelligent Transportation Systems, 2015, 16(6): 3017-3032.

[2] FLAD M, FROHLICH L, HOHMANN S. Cooperative shared control driver assistance systems based on motion primitives and differential games[J]. IEEE Transactions on Human-Machine Systems， 2017，47(5): 711-722.

[3] SOUALMI B, SENTOUH C, POPIEUL J C, et al. Automation-driver cooperative driving in presence of undetected obstacles[J]. Control Engineering Practice, 2014, 24: 106-119.

[4] ANDERSOM S J, KARUMANCHI S B, IAGNEMMA K, et al. The intelligent copilot: A constraint-based approach to shared-adaptive control of ground vehicles[J]. IEEE Intelligent Transportation Systems Magazine， 2013，5(2): 45-54.

[5] 谈东奎. 人机共享的驾驶员横向辅助系统关键技术研 究[D]. 合肥: 合肥工业大学, 2017 .

TAN Dongkui. Research on key technologies of humanmachine shared lateral driver assistance system[D]. Hefei: Hefei University of Technology, 2017.

[6] 赵治国, 周良杰, 朱强. 无人驾驶车辆路径跟踪控制预 
瞄距离自适应优化[J]. 机械工程学报, 2018, 54(24): 166-173.

ZHAO Zhiguo, ZHOU Liangjie, ZHU Qiang. Preview distance adaptive optimization for the path tracking control of unmanned vehicle[J]. Journal of Mechanical Engineering, 2018, 54(24): 166-173.

[7] GROOT S D, RICOTE F C, WINTER J C F D. The effect of tire grip on learning driving skill and driving style: A driving simulator study[J]. Transportation Research Part F: Psychology \& Behaviour, 2012， 15(4): 413-426.

[8] SUN Bohua, DENG Weriwen, WU Jian, et al. Analysis of coupling mechanism between driving skill and driving style in driver personalization[C]// The 14th International Symposium on Advanced Vehicle Control, July 16-29, 2018, Beijing International Convention Center, Beijing. Beijing: AVEC, 2018: WeB 1-4.

[9] MARTINEZ C M, HEUKE M, WANG Feiyue, et al. Driving style recognition for intelligent vehicle control and advanced driver assistance: A survey[J]. IEEE Transactions on Intelligent Transportation Systems, 2017, 19(3): 666-676.

[10] MARS F, CHEVREL P. Modelling human control of steering for the design of advanced driver assistance systems[J]. Annual Reviews in Control, 2017, 44 : 292-302.

[11] 许力, 胡杰, 孟武强. 个性化驾驶员模型及其在车辆测 试中应用[J]. 机械工程学报，2015，51(18): 136-142. XU Li, HU Jie, MENG Wuqiang. Personalized driver model and its application to vehicle testing[J]. Journal of Mechanical Engineering, 2015，51(18): 136-142.

[12] ZAVALKO A. Applying energy approach in the evaluation of eco-driving skill and eco-driving training of truck drivers[J]. Transportation Research Part D: Transport \& Environment, 2018, 62: 18-26.

[13] 刘明周, 蒋倩男, 扈静. 基于面部几何特征及手部运动 特征的驾驶员疲劳检测 [J]. 机械工程学报, 2019, 55(2): 18-26.

LIU Mingzhou, JIANG Qiannan, HU Jing. Based on facial geometric features and hand motion characteristics driver fatigue detection[J]. Journal of Mechanical Engineering, 2019, 55(2): 18-26.

[14] HOOGENDOORN S P, BOVY P H L. State-of-the-art of vehicular traffic flow modeling $[\mathrm{J}]$. Proceedings of the Institution of Mechanical Engineers Part I Journal of Systems and Control Engineering, 2001, 215: 283-303.
[15] ENRIQUE C, GRANDE ZACARIAS, AIDA C, et al. A state of theart review of the sensor location, flow observability, estimation, and prediction problems in traffic networks[J]. Journal of Sensors, 2015, 2015: 1-26.

[16] ZHANG Yicai, XUE Yu, ZHANG Peng, et al. Bifurcation analysis of traffic flow through an improved car-following model considering the time-delayed velocity difference[J]. Physica A: Statistical Mechanics and its Applications, 2019, 514: 133-140.

[17] HUANG Wenhao, SONG Guojie, HONG Haikun, et al. Deep architecture for traffic flow prediction: Deep belief networks with multitask learning[J]. IEEE Transactions on Intelligent Transportation Systems, 2014, 15(5): 2191-2201.

[18] BORSCHE R, MEURER A. Microscopic and macroscopic models for coupled car traffic and pedestrian flow[J]. Journal of Computational and Applied Mathematics, 2018: 356-382.

[19] 王建强, 吴剑, 李洋. 基于人-车-路协同的行车风险场 概念、原理及建模 [J]. 中国公路学报，2016，29(1): 105-114.

WANG Jianqiang, WU Jian, LI Yang. Concept, princople and modeling of driving risk field based on drivervehicle-road interaction[J]. China Journal of Highway and Transport, 2016, 29(1): 105-114.

[20] BERND F. A growing neural gas network learns topologies[J]. Advances in Neural Information Processing Systems, 1995, 7: 625-632.

[21] 陈佩. 主成分分析法研究及其在特征提取中的应用 $[D]$. 西安：陕西师范大学，2014.

CHEN Pei. Study on principal component analysis and its application in feature extraction[D]. Xi'an: Shaanxi Normal University, 2014.

[22] ÖZKAN T, LAJUNEN T. What causes the differences in driving between young men and women? The effects of gender roles and sex on young drivers' driving behaviour and self-assessment of skills[J]. Transportation Research Part F: Psychology \& Behaviour, 2006, 9(4): 269-277.

[23] LAWRENCE R R. A tutorial on hidden markov models and selected applications in speech recognition[J]. Proceeding of the IEEE, 1990， 77(2): 267-296.

[24] REYNOLDS D A, ROSE R C. Robust text-independent speaker identification using Gaussian mixture speaker models[J]. IEEE Trans. Speech \& Audio Processing, 1995, 
3(1): 72-83.

[25] NAGEL K, SCHRECKENBERG M. A cellular automaton model for freeway traffic[J]. Journal de Physique I, 1992, 2(12): 2221-2229.

作者简介: 孙博华, 男, 1988 年出生, 博士研究生。主要研究方向为基 于人性化和个性化的汽车智能辅助驾驶及自主操纵。

E-mail: sunbh14@mails.jlu.edu.cn
邓伟文(通信作者), 男, 1963 年出生, 博士, 教授, 博士研究生导师。 主要研究方向为基于人性化和个性化的汽车智能辅助驾驶及自主操纵。 E-mail: kwdeng@188.com

吴坚, 男, 1977 年出生, 博士, 教授, 博士研究生导师。主要研究方向 为汽车先进电控、整体优化和集成控制。

E-mail: wujian@jlu.edu.cn

李雅欣, 女, 1992 年出生, 博士研究生。主要研究方向为面向汽车虚拟 测试的传感器建模和越野条件下自动驾驶关键问题。

E-mail: liyaxin0930@foxmail.com 